
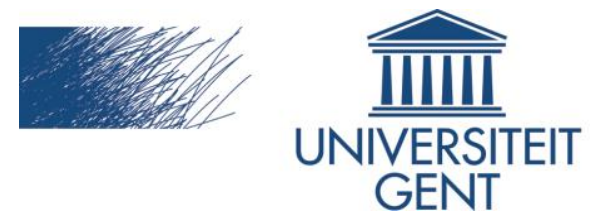

biblio.ugent.be

The UGent Institutional Repository is the electronic archiving and dissemination platform for all UGent research publications. Ghent University has implemented a mandate stipulating that all academic publications of UGent researchers should be deposited and archived in this repository. Except for items where current copyright restrictions apply, these papers are available in Open Access.

This item is the archived peer-reviewed author-version of: Carbon quantum dots as a dual platform for the inhibition and light-based destruction of collagen fibers: implications for the treatment of eye floaters

Authors: Alexandre Barras, Felix Sauvage, Ines de Hoon, Kevin Braeckmans, Dawei Hua, Gaetan Buvat, Juan C. Fraire, Christophe Lethien, J. Sebag, Michael Harrington, Amar Abderrahmani, Rabah Boukherroub, Stefaan De Smedt, Sabine Szunerits

In: Nanoscale Horizons 6(6): 449-461

To refer to or to cite this work, please use the citation to the published version:

Alexandre Barras, Felix Sauvage, Ines de Hoon, Kevin Braeckmans, Dawei Hua, Gaetan Buvat, Juan C. Fraire, Christophe Lethien, J. Sebag, Michael Harrington, Amar Abderrahmani, Rabah Boukherroub, Stefaan De Smedt, Sabine Szunerits (2021)

Carbon quantum dots as a dual platform for the inhibition and light-based destruction of collagen fibers: implications for the treatment of eye floaters

Nanoscale Horizons 6(6): 449-461

DOI: 10.1039/d1nh00157d 


\title{
Carbon quantum dots as a dual platform for the inhibition and light-based destruction of collagen fibers: implications for the treatment of eye floaters
}

\author{
Alexandre Barras, ${ }^{1 * *}$ Félix Sauvage, ${ }^{2 * *}$ Inès de Hoon, ${ }^{1,2}$ Kevin Braeckmans, ${ }^{2}$ Dawei Hua, ${ }^{2}$ \\ Gaëtan Buvat, ${ }^{1}$ Juan C. Fraire, ${ }^{2}$ Christophe Lethien, ${ }^{1}$ J. Sebag, ${ }^{3,4}$, Michael Harrington ${ }^{5}$, Amar \\ Abderrahmani, ${ }^{1}$ Rabah Boukherroub, ${ }^{1}$ Stefaan De Smedt, ${ }^{2 *}$ Sabine Szunerits ${ }^{1 *}$ \\ ${ }^{1}$ Univ. Lille, CNRS, Centrale Lille, Univ. Polytechnique Hauts-de-France, UMR 8520 - \\ IEMN, F-59000 Lille, France \\ ${ }^{2}$ Laboratory of General Biochemistry and Physical Pharmacy, Faculty of Pharmaceutical \\ Sciences, Ghent University, 9000 Ghent, Belgium. \\ ${ }^{3}$ VMR Institute for Vitreous Macula Retina, Huntington Beach, California 92647, United \\ States \\ ${ }^{4}$ Doheny Eye Institute/UCLA, Los Angeles, California 90033, United States \\ ${ }^{5}$ Huntington Memorial Research Institute, Pasadena, CA, USA
}

\begin{abstract}
Common in myopia and aging, vitreous opacities arise from clumped collagen fibers within the vitreous body that cast shadows on the retina, appearing as 'floaters' to the patient. Vitreous opacities degrade contrast sensitivity function and can cause significant impairment in visionrelated quality-of-life, representing an unmet and underestimated medical need. One therapeutic approach could be the use of versatile light-responsive nanostructures which (i) interfere with the formation of collagen fibers and /or (ii) destroy aggregates of vitreous collagen upon pulsed-laser irradiation at low fluences. In this work, the potential of positively and negatively charged carbon quantum dots (CQDs) to interfere with the aggregation of type I collagen is investigated. We demonstrate that fibrillation of collagen I is prevented most strongly by positively charged CQDs (CQDs-2) and that pulsed-laser illumination allowed to destroy type I collagen aggregates and vitreous opacities (as obtained from patients after vitrectomy) treated with CQDs-2.
\end{abstract}

\footnotetext{
**equal contribution

*To whom correspondence should be send to: Stefaan.Desmedt@UGent.be or sabine.szunerits@univlille.fr (SS)
} 
Keywords: Vitreous floaters, carbon quantum dots (CQDs), collagen fibers, pulsed-laser, ophthalmology, fibrillation. 


\section{Introduction}

Opacities in the vitreous body are one of the most common causes of severe impairment in ocular vision. Vitreous opacities are characterized by floating objects inside the vitreous body, which can result from several origins including myopia, infection, diabetic retinopathy, aging and eye injury. Vitreous is a highly hydrated gel-like structure ( $>98 \%$ water), which is maintained by a diluted network of long, thin collagen fibrils. Individual collagen fibrils are normally spaced apart by hyaluronan, an anionic, non-sulfated glycosaminoglycan (polysaccharide), which attracts water and generates a swelling pressure that inflates the gel. Vitreous collagen fibrils are heterotypic in composition, containing type IX collagen being regularly distributed along the fibril surfaces $;{ }^{1}$ rope-like fibrils of collagen types II surrounding a central core of hybrid V/XI, with type II collagen predominating. ${ }^{2}$ Type IX collagen has chondroitin sulfate glycosaminoglycan chains attached to it which extend away from the fibril surfaces and space apart the collagen fibrils, thereby preventing fibril aggregation. ${ }^{3}$ Vitreous opacities and the degeneration of the vitreous body result from several interconnected mechanisms including the progressive cross-linking of individual collagen fibrils and their aggregation, and finally, liquefaction. ${ }^{4}$ Liquefaction is a physicochemical degenerative change that disrupts the homogeneity of the gel vitreous and is characterized by the dissociation of hyaluronan from collagen. Thus, vitreous progressively liquefies as the collagen fibril aggregates and HA dissociates from collagen drawing water with it to form liquid vitreous. Collagen becomes concentrated in some areas of the gel, while other parts of the vitreous body are devoid of collagen fibrils forming liquid compartments. ${ }^{5}$ Another proposed mechanism for vitreous liquefaction is that collagen fibrils are fragmented. ${ }^{6}$ It is believed that type IX collagen shields type II collagen and prevents fusion between fibrils. ${ }^{3}$ Loss of type IX collagen results in type II collagen becoming increasingly exposed, predisposing to fusion of adjacent fibrils on contact. In both cases, when the aggregates are sufficiently large, they are visible as vitreous opacities resulting in degradation of contrast sensitivity function ${ }^{4,7}$ with a negative impact on the quality of life. ${ }^{8,9}$ Other mechanisms of vitreous liquefication can be considered, including enzymatic liquefaction and oxidative stress-induced liquefaction. ${ }^{10}$

The treatment of vitreous opacification relies on its origin. However, in case of treatment failure, the only curative treatment for removing floaters remains vitrectomy (i.e. a surgical removal of vitreous through small openings in the pars plana) ${ }^{11,12}$ or by Nd:YAG laser treatment. ${ }^{13}$ However, treatment with the Nd:YAG laser has relatively little efficacy ${ }^{14}$ and sometimes can worsen the eye symptoms. In addition, if the opacities are located too close to the retina, for 
safety reasons, laser treatment cannot be achieved. There is a true medical need for searching for safe and non-invasive methods for treating vitreous floaters. Also, non-surgical therapies have been under development, but, pharmacologic vitreolysis has not been designed to address vitreous opacities. ${ }^{10,15,16}$ More recently, the use of light absorbing gold nanoparticles (Au NPs) was proposed by some of us as an alternative solution. ${ }^{17}$ When coated with hyaluronic acid, after injection in vitreous ex vivo, these particles diffuse through vitreous and cluster on the collagenous opacities. Thanks to their photothermal properties, Au NPs heat up in an ultrafast manner when illuminated by a nanosecond pulsed-laser; this leads to the evaporation of the water surrounding the Au NPs and the formation of vapor nanobubbles (VNBs). We showed that VNBs collapsing at the surface of the vitreous opacities provide sufficient mechanical force to ablate them. Importantly, the combined use of the Au NPs and nanosecond laser pulses allowed destruction of the opacities at a laser energy 1000 times lower than typically used in YAG laser therapy. Of note is that pulsed lasers are commonly used in ophthalmology for the treatment of cataract and in corneal surgery.

Carbon-based nanostructures, such as carbon quantum dots (CQDs), offer promising therapeutic potential as they can engage with biological molecules. ${ }^{18}$ These nanostructures have proven to be excellent drug carrier due to its good biocompatibility. ${ }^{19}$ The easy of introducing functional groups has enabled their application as medical countermeasure to enveloped viruses, including next to herpes simplex virus type $1^{20}$ human cornaiviruses. The intrinsic fluoresce properties make them well adapted for bio-imaging but allows as well its use in biosensing platforms. ${ }^{18}$ One of their applications is the treatment of eye infections with highly positively charged CQDs. ${ }^{21}$ CQDs have also been explored for the treatment of corneal neovascularization ${ }^{22}$ and to improve retinal angiography. ${ }^{23,24}$ Motivated by the reported ability of CQDs to prevent human insulin fibrillation, ${ }^{25-28}$ and the potential of CQDs in regulating the aggregation behavior of human islet amyloid polypeptide (hIAPP) ${ }^{29}$ and by their good light absorbing properties over a wide wavelength spectrum, we aim to investigate (i) the potential of CQDs to inhibit the formation of collagen aggregates and (ii) the combined use of CQDs and pulsed laser light to destroy collagen fibers and vitreous opacities through the generation of VNBs (Figure 1). 


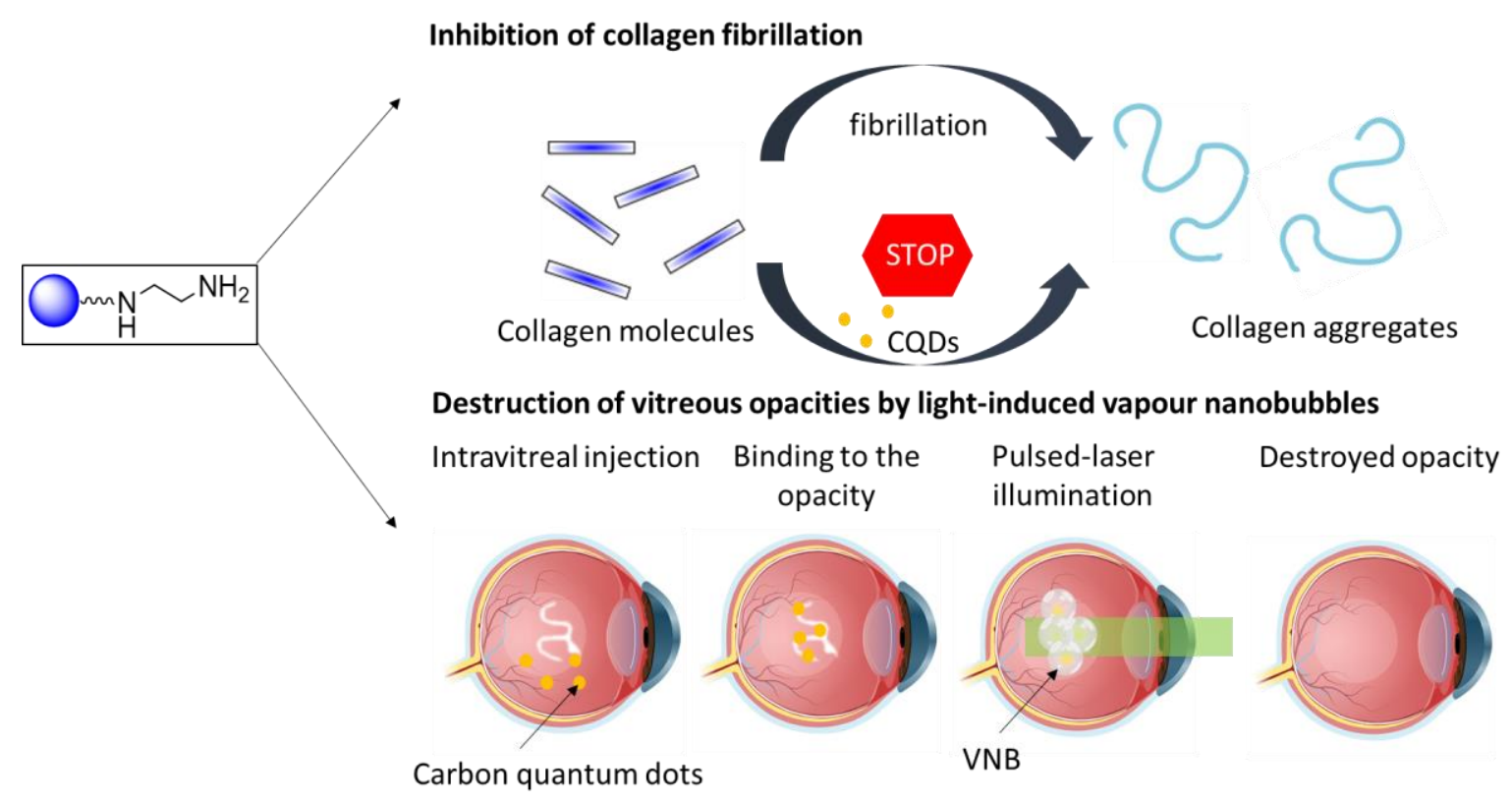

Figure 1. Carbon quantum dots (CQDs) to inhibit the formation of collagen aggregates (top) and to destroy collagen aggregates by light induced VNBs (bottom).

\section{Results and discussion}

\subsection{One-step microwave-assisted synthesis of aminated and carboxylated fluorescent} carbon dots

We have shown previously that a large panel of differently functionalized CQDs can be produced by hydrothermal carbonization of different organic precursors. ${ }^{30}$ Hydrothermal techniques lead to highly fluorescent CQDs, but long reaction times and high temperature conditions using a close Teflon-line reactor lead too often to a lack of reproducibility and replicability. Alternatively, microwave-assisted synthesis of CQDs is a more reproducible and easier approach due to the on-line monitoring of temperature and pressure in the reaction vessels. $^{18,31}$ To this end, a rapid synthesis of carboxylic acid-decorated CQDs (CQD-1) using glucosamine as a starting material and an amino acid ( $\beta$-alanine) as surface a passivating agent was investigated. ${ }^{32}$ Aminated CQDs (CQD-2) were prepared in a comparable manner from glucosamine hydrochloride and ethylenediamine as a passivating agent (Figure 2a). ${ }^{33}$ To remove larger precipitates, the CQDs suspension was centrifuged and then dialyzed against water with a final yield of $0.8 \%$ and $3.4 \%$ for CQD-1 and CQD-2, respectively. CQD-1 exhibit a spherical shape with a primary average diameter of $20.8 \pm 6.6 \mathrm{~nm}$ (Table 1), as determined by AFM (Figure 2b). CQD-1 form a "bunch of grapes" in solution and their size reaches up to 50-350 $\mathrm{nm}$ in good accordance with the hydrodynamic size recorded by DLS. CQD-2 display a spherical shape with an average diameter of $16.8 \pm 6.7 \mathrm{~nm}$ (Table 1), as determined by AFM 
(Figure 2b). Transmission electron microscopy (TEM) observations reveals that the CQDs were almost spherical with an average particle size of $\sim 26$ and $\sim 19 \mathrm{~nm}$, respectively, in a good concordance with sizes determined by AFM (Figure S1). The amorphous nature of the CQDs was concluded by the absence of clear lattice fringes as well as any clear pattern in the selected area electron diffraction in the TEM microscopy (data not shown).

Table 1: Physico-chemical properties of CQDs.

\begin{tabular}{|l|l|l|}
\hline & CQD-1 & CQD-2 \\
\hline$\zeta / \mathrm{mV}$ & $(-) 24.0 \pm 1.6$ & $(+) 32.5 \pm 0.8$ \\
\hline Size determined by AFM / nm & $20.8 \pm 6.6$ & $16.8 \pm 6.7$ \\
\hline Hydrodynamic size (intensity)/ nm & $389 \pm 123(100 \%)$ & $\begin{array}{l}10.6 \pm 2.3(63 \%) \\
265 \pm 199(29 \%)\end{array}$ \\
\hline $\mathrm{O} /$ at. \% & 22.8 & 14.4 \\
\hline $\mathrm{N} /$ at. \% & 5.7 & 13.7 \\
\hline $\mathrm{I}_{\mathrm{D}} / \mathrm{I}_{\mathrm{G}}$ ratio & 1.94 & 1.56 \\
\hline Colloidal stability in water & excellent & excellent \\
\hline Colloidal stability in DMEM & excellent & excellent \\
\hline Quantum yield $(\Phi)$ & 0.008 & 0.002 \\
\hline
\end{tabular}

DMEM = Dulbecco's Modified Eagle's

The chemical composition of the carbon dots was assessed by X-ray photoelectron spectroscopy (XPS, see survey in SI, Figure S2). The $\mathrm{C}_{1 \mathrm{~s}}$ high resolution XPS spectra of the CQDs depict different carbon features (Figure 2c). In the case of CQD-1, the high-resolution spectrum of the $\mathrm{C}_{1 \mathrm{~s}}$ reveals the presence of fives peaks ascribed to $\mathrm{C}=\mathrm{C}(283.8 \mathrm{eV}), \mathrm{C}-\mathrm{C} / \mathrm{C}-\mathrm{H}(285.0 \mathrm{eV})$, $\mathrm{C}-\mathrm{N} / \mathrm{C}-\mathrm{O}(286.8 \mathrm{eV}), \mathrm{C}=\mathrm{O}(287.9 \mathrm{eV})$ and $-\mathrm{O}-\mathrm{C}=\mathrm{O}(289.2 \mathrm{eV})$. The presence of carbonyl and carboxylic functions is additionally validated by the presence of a band at $530.7 \mathrm{eV}(\mathrm{C}=\mathrm{O})$ in the $\mathrm{O}_{1 \mathrm{~s}}$ high resolution spectrum, next to $531.5 \mathrm{eV}(\mathrm{C}-\mathrm{O})$. The $\mathrm{N}_{1 \mathrm{~s}}$ high resolution spectrum can be curve-fitted with bands at $398.3 \mathrm{eV}$ (pyridinic $\mathrm{C}=\mathrm{N}-\mathrm{C}$ ), $399.1 \mathrm{eV}$ (pyrrolic N-H) and $400.3 \mathrm{eV}$ (graphite-like structure $\mathrm{N}_{-} \mathrm{C}_{3}$ ). In case of CQD-2 (Figure 2d), the high-resolution spectrum of the $\mathrm{C}_{1 \mathrm{~s}}$ can be deconvoluted with fives peaks assigned to $\mathrm{C}=\mathrm{C}(283.9 \mathrm{eV}), \mathrm{C}-\mathrm{C} / \mathrm{C}-$ $\mathrm{H}(285.0 \mathrm{eV}), \mathrm{C}-\mathrm{N} / \mathrm{C}-\mathrm{O}(286.1 \mathrm{eV}), \mathrm{C}=\mathrm{O}(287.1 \mathrm{eV})$ and $-\mathrm{O}-\mathrm{C}=\mathrm{O}(288.4 \mathrm{eV})$. The presence of oxygen functions is also corroborated by the presence of the band at $530.3 \mathrm{eV}(\mathrm{C}=\mathrm{O})$ in the $\mathrm{O}_{1 \mathrm{~s}}$ high resolution spectrum, next to $531.6 \mathrm{eV}(\mathrm{C}-\mathrm{O})$. The $\mathrm{N}_{1 \mathrm{~s}}$ high resolution spectrum shows comparable bands as CQDs-1: $398.3 \mathrm{eV}$ (pyridinic $\mathrm{C}=\mathrm{N}-\mathrm{C}$ ), $399.2 \mathrm{eV}$ (pyrrolic N-H) and 400.9 eV (graphite-like structure $\mathrm{N}-\mathrm{C}_{3}$ ). Both CQDs have a variety of polar groups (hydroxyl, carbonyl, carboxyl, amine) present on their surface. The presence of $\mathrm{N}-\mathrm{C}_{3}$ moieties indicates that some nitrogen atoms were incorporated into the carbon framework. The oxygen content in 
the CQD-1 and CQD-2 was determined to be 22.8 at. \% and 14.4 at. \%, respectively. Higher nitrogen content (13.7 at. \%) was found in CQD-2 in comparison with CQD-1 (5.7 at. \%). Furthermore, the presence of primary amino groups was validated by the ninhydrin reaction test (see SI, Figure S3), which allowed estimating a surface coverage of $0.24 \pm 0.2 \mathrm{mmol} \mathrm{g}^{-1}$.

The chemical composition of the CQDs was further investigated by Fourier transform infrared (FT-IR) spectroscopy (Figure 2e). The FTIR spectrum of CQD-2 comprises a broad band between $3415-3425 \mathrm{~cm}^{-1}$ attributed to the $\mathrm{O}-\mathrm{H} / \mathrm{N}-\mathrm{H}$ stretching vibrations, while the $\mathrm{C}-\mathrm{H}$ stretching and bending vibrations are located at 2932 and at $1384 \mathrm{~cm}^{-1}$, respectively. The band at $1636 \mathrm{~cm}^{-1}$ is due to $\mathrm{C}=\mathrm{N}$ and $\mathrm{C}=\mathrm{C}$ bonds of the aromatic structure, and $\mathrm{C}=\mathrm{O}$ stretching vibration (-NHCO-). Compared to CQD-2, the more intense peaks at 1083 and $1041 \mathrm{~cm}^{-1}$ in the FTIR spectrum of CQD-1 are associated with C-O stretching of either the residual carbohydrate or $\beta$-alanine. The sharp peak at $1710 \mathrm{~cm}^{-1}$ is assigned to the asymmetric stretching vibration of carboxylic acid moieties. $^{34}$

Typical Raman spectra of CQD-1 and CQD-2 are seen in Figure 2f. The observed peaks are typical of $\mathrm{sp}^{2}$ carbon materials at $1580 \mathrm{~cm}^{-1}$ (G-band) and $1378 \mathrm{~cm}^{-1}$ (D band), indicating that the material is ordered and graphitic in nature. The fact that the D band is rather broad points towards a disordered structure, keeping in mind that the D-peak is not related to disorder but indirectly to the size of the $\mathrm{sp}^{2}$ coordinating ring domains. The $\mathrm{I}_{\mathrm{D}} / \mathrm{I}_{\mathrm{G}}$ ratio is 1.94 for CQD-1 and 1.56 for CQD-2 suggesting large surface disorder and amorphous nature. ${ }^{35}$

Zeta potential analysis is a source of information in terms of surface functionalities (Figure S4a). CQD-1 display a negative zeta potential value $(\zeta=-24.0 \pm 1.6 \mathrm{mV})$ in pure milli-Q water. The negative surface charge is due to an abundance of deprotonated carboxyl groups on its surface. In contrast, CQD-2 exhibits a positive surface charge with $\zeta=+32.5 \pm 0.8 \mathrm{mV}$, underlining the presence of $\mathrm{NH}_{3}{ }^{+}$groups. Due to the presence of hydrophilic groups, CQDs were readily dispersible at high concentrations $\left(>10 \mathrm{mg} \mathrm{mL}^{-1}\right)$ in pure water or PBS. The dispersion of $250 \mu \mathrm{g} \mathrm{mL}^{-1}$ CQDs (Figure S4b) in pure water and Dulbecco's Modified Eagle's medium (DMEM) is excellent for both particles. In PBS, the colloidal stability of CQD-2 is reduced and a precipitate is formed after $1 \mathrm{~h}$ incubation at $37{ }^{\circ} \mathrm{C}$. 
(a)

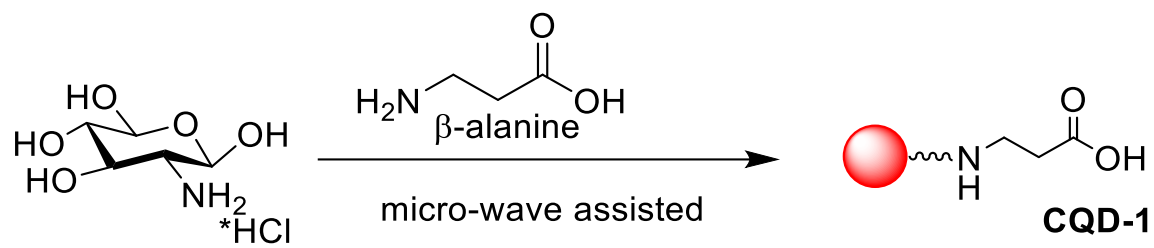

glucosamine

hydrochloride

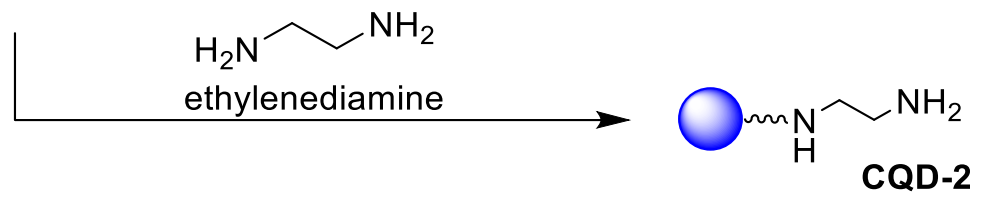

(b)
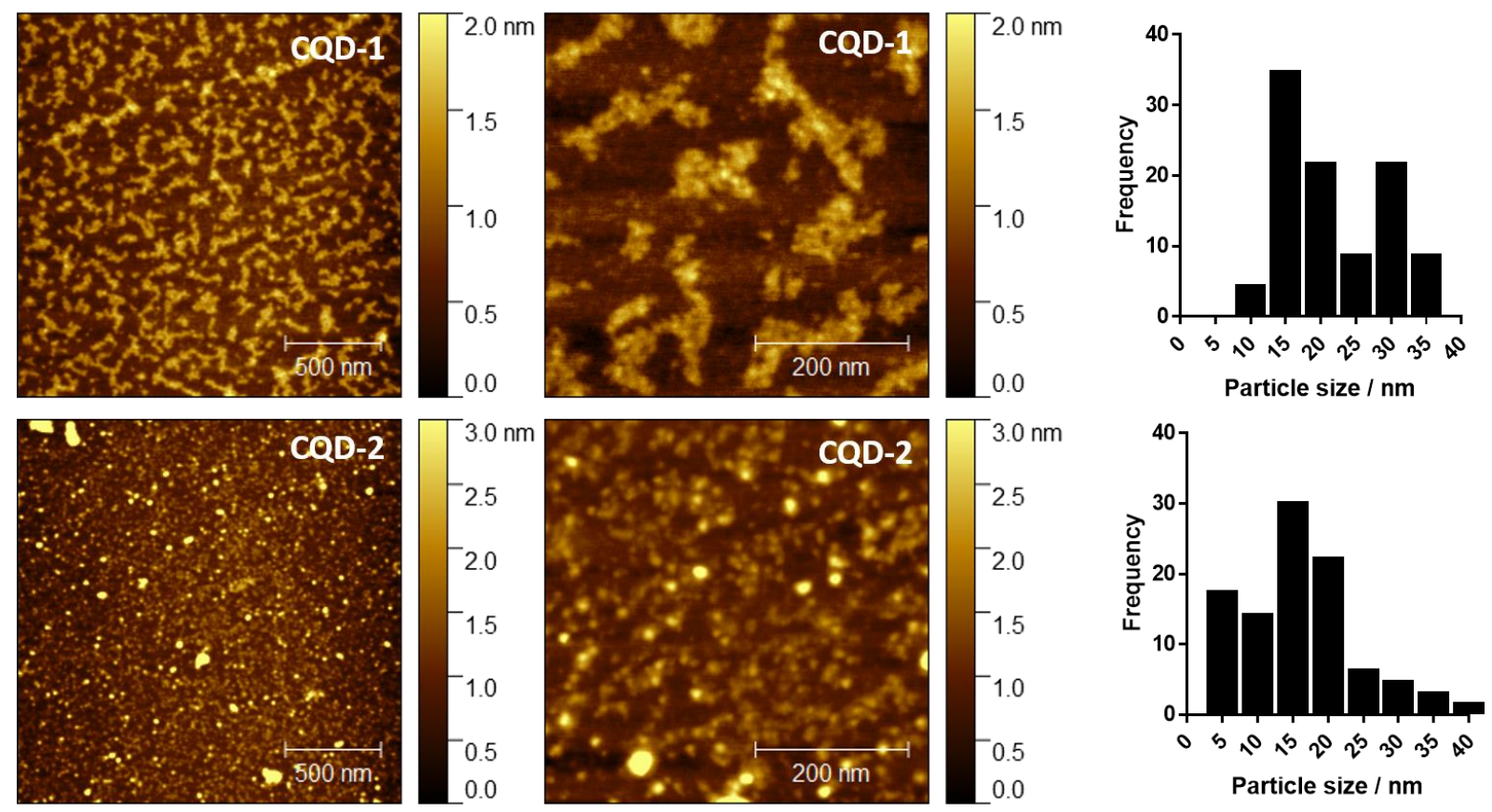

(c)
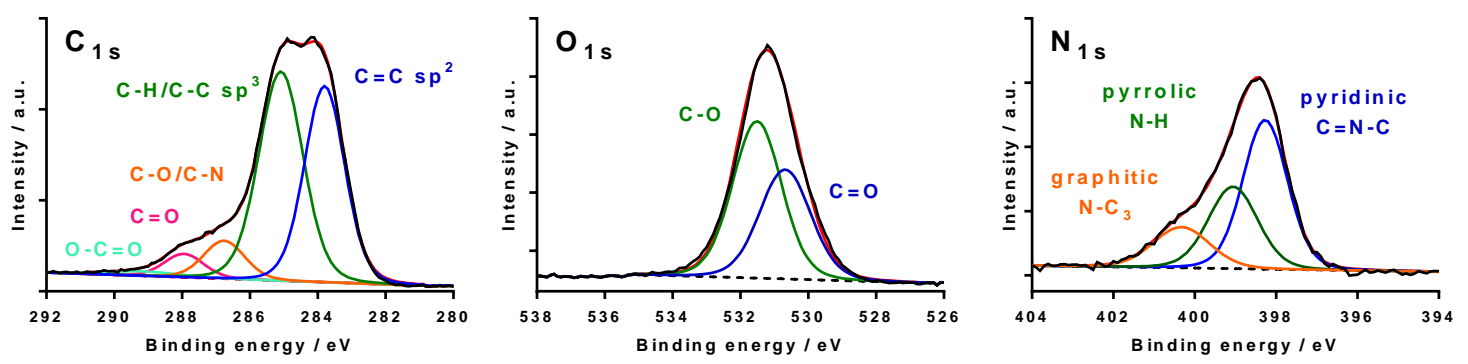

(d) 


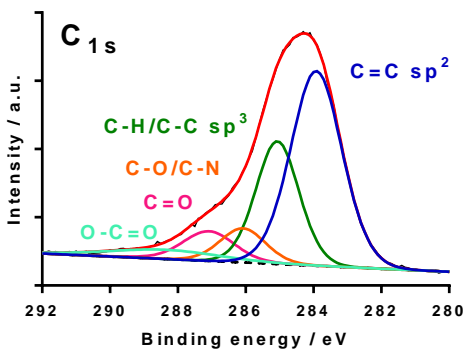

(e)

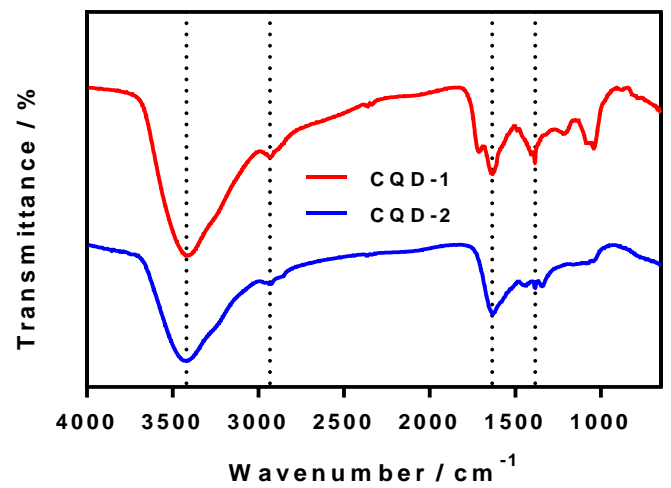

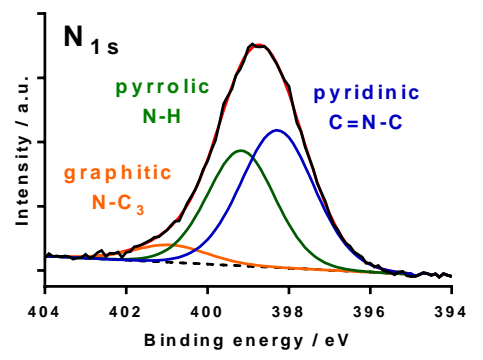

(f)

Figure 2: (a) Schematic representation of the synthesis of CQD-1 and CQD-2. (b) AFM images of CQD-1 and CQD-2 together with size distribution histograms. (c) XPS analysis of CQD-1: $\mathrm{C}_{1 \mathrm{~s}}, \mathrm{O}_{1 \mathrm{~s}}$ and $\mathrm{N}_{1 \mathrm{~s}}$. (d) XPS analysis of CQD-2: $\mathrm{C}_{1 \mathrm{~s}}, \mathrm{O}_{1 \mathrm{~s}}$ and $\mathrm{N}_{1 \mathrm{~s}}$. (e) FT-IR spectra of CQD-1 (red) and CQD-2 (blue). (f) Raman spectra of CQD-1 (red) and CQDs-2 (blue).

The CQDs absorb in the short-wavelength region with a maximum at around $220 \mathrm{~nm}$ due to $\pi$ $\pi^{*}$ transition of $\mathrm{C}=\mathrm{C}$ and $\mathrm{C}=\mathrm{N}$ carbon cores with a weak extending tail in the visible region (Figure 3a). An additional shoulder at approximatively $280 \mathrm{~nm}$ is attributed to $\pi-\pi^{*}$ transition of different $\mathrm{sp}^{2}$ domains. ${ }^{36}$ The absorption shoulder at around $306 \mathrm{~nm}$ in CQD-1 is due to $\mathrm{n}-\pi^{*}$ transitions of $\mathrm{C}=\mathrm{O}$. This contribution is decreased in CQD-2 due to the high content of ethylenediamine $;{ }^{37}$ the strong absorption extending from 350 to $600 \mathrm{~nm}$ is related to surface passivation of CQDs-2 with ethylenediamine. ${ }^{38}$

As expected, CQD-1 and CQD-2 reveal photoluminescence characteristic (Figure 3b) with a maximum fluorescence intensity at $450 \mathrm{~nm}$ under $380 \mathrm{~nm}$ excitation. ${ }^{39}$ The fluorescence emission is wavelength-dependent (Figure 3c, 3d). CQD-1 display a broad (full width at half maximum of around $100 \mathrm{~nm}$ ) and intense photon emission, while CQD-2 exhibit a lower fluorescence. Increasing the excitation wavelength to $500 \mathrm{~nm}$ results in a red-shift of the emission band to $590 \mathrm{~nm}$ for CQD-1 and $580 \mathrm{~nm}$ for CQD-2, with the fluorescence emission 
intensity of CQD-1 being somehow higher than that of CQDs-2. Using quinine sulfate as reference with a quantum yield of 0.54 , the fluorescent quantum yields of CDQ-1 and CQD-2 varied between 0.008 and 0.002, respectively (Table 1, Figure S5). The long storage stability at $4{ }^{\circ} \mathrm{C}$ and effects of parameters such as prolonged UV exposure of CQDs were studied. The fluorescence intensity of CQD-1 had no obvious decrease (14\%) after being stored 2-years at $4{ }^{\circ} \mathrm{C}$ (Figure S6). The fluorescence intensity of CQD-2 had higher decrease (48\%). In contrast, the normalized fluorescence intensity before and after 2-years for both CQDs was almost unchanged, which suggest no chemical modifications. CQDs displayed UV-dependent fluorescence behavior. CQD-2 present a relatively stable fluorescence emission intensity during 30 min exposure to UV irradiation $\left(360 \mathrm{~nm}, 10 \mathrm{~mW} \mathrm{~cm}^{-2}\right)$ in comparison to CQD-1.

(a)

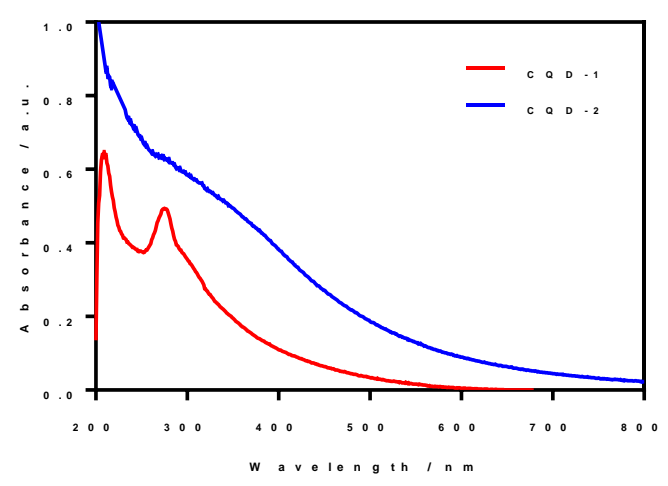

(c)

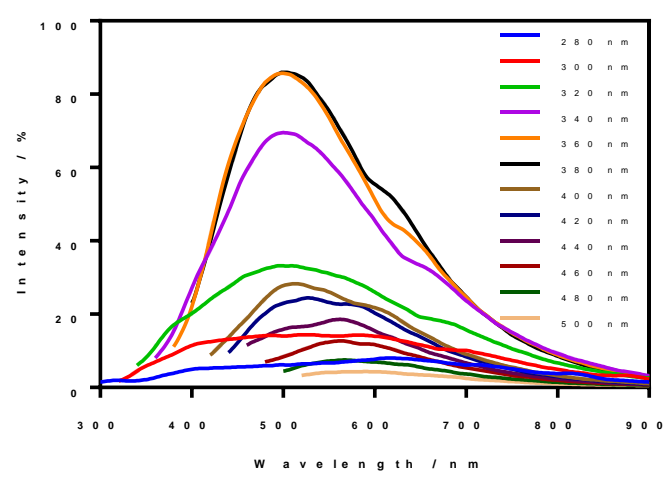

(b)

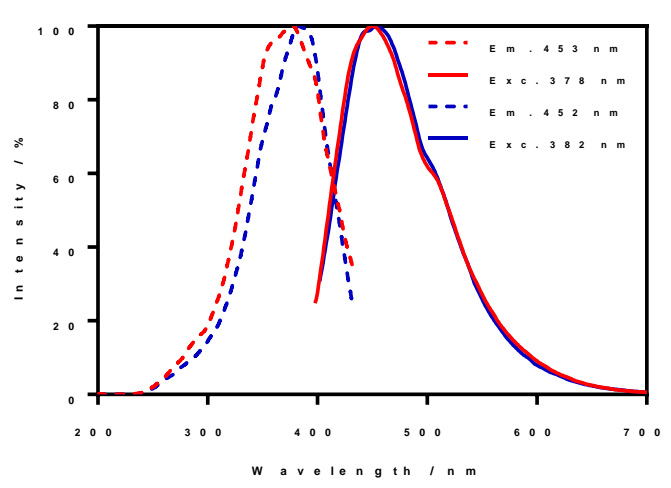

(d)

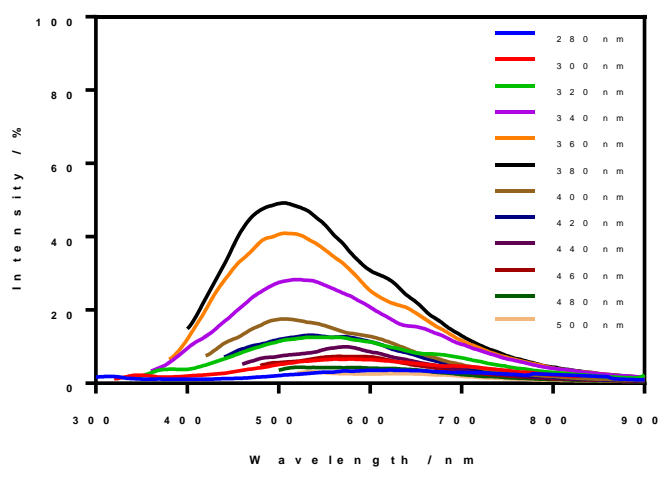

Figure 3: (a) UV-vis spectra of CQD-1 and CQD-2 at $20 \mu \mathrm{g} \mathrm{mL}^{-1}$ in PBS (pH 7.4, $0.01 \mathrm{M}$ ). (b) Normalized emission (exc. at $378 \mathrm{~nm}$ ) and normalized excitation (em. $453 \mathrm{~nm}$ ) spectra of CDQ1; normalized emission (exc. at $382 \mathrm{~nm}$ ) and normalized excitation (em. $452 \mathrm{~nm}$ ) spectra of CQD-2. (c) Emission spectra of CQD-1 at progressively increasing excitation wavelengths from 280 to $500 \mathrm{~nm}$. (d) Emission spectra of CQD-2 at progressively increasing excitation wavelengths from 280 to $500 \mathrm{~nm}$. 


\subsection{Photothermal properties of CQD-1 and CQD-2}

The photothermal properties of CQD-1 and CQD-2 (50 $\left.\mu \mathrm{g} \mathrm{mL}^{-1}\right)$ were determined upon irradiation the aqueous solutions with a laser at $810 \mathrm{~nm}$ for $15 \mathrm{~min}$. Figure 4 indicates that in both cases a temperature plateau is reached after $10 \mathrm{~min}$, reaching about $50^{\circ} \mathrm{C}$ for a laser power of $1 \mathrm{~W} \mathrm{~cm}$ and $66{ }^{\circ} \mathrm{C}$ for $2 \mathrm{~W} \mathrm{~cm}^{-2}$. However, the same temperature trend is observed with PBS solution, indicating poor photothermal properties of these CQDs under these experimental conditions.

(a)

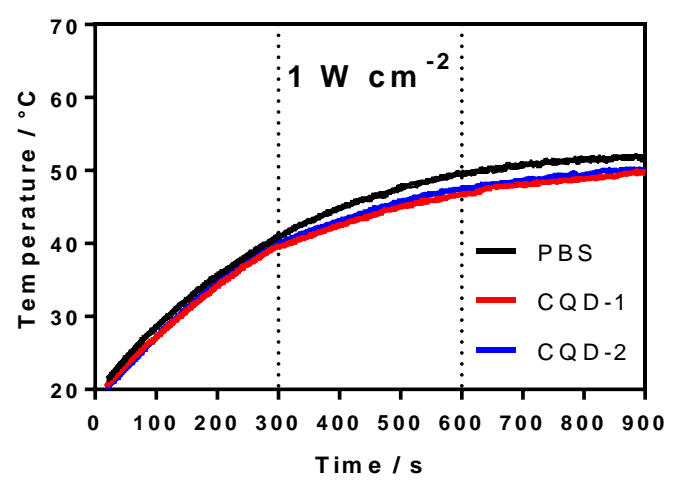

(b)

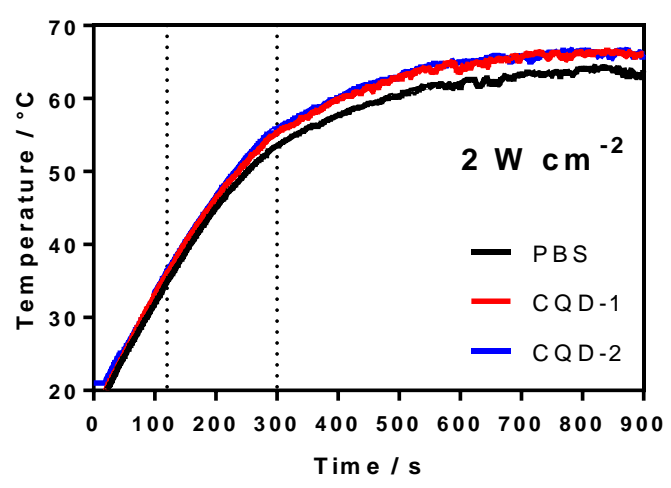

Figure 4: Photothermal properties of CQDs. Temperature profiles of CQDs $\left(50 \mu \mathrm{g} \mathrm{mL}^{-1}\right)$ upon laser irradiation at $810 \mathrm{~nm}$ laser at two different power densities: $1 \mathrm{~W} \mathrm{~cm}^{-2}$ (a) and $2 \mathrm{~W}$ $\mathrm{cm}^{-2}(\mathrm{~b})$.

\subsection{Metabolic activity and cellular uptake of CQDs}

The cell toxicity of CQDs-1 and CQDs-2 was established on ARPE-19 and MIO-M1 cell lines after $8 \mathrm{~h}$ and $24 \mathrm{~h}$ incubation (Figure 5). The CQDs toxicity was evaluated using the resazurin assay, based on the conversion of non-fluorescent dye to a fluorescent molecule by mitochondrial and cytosolic enzymes. All CQDs are non-toxic to ARPE-19 and MIO-M1 cells until $100 \mu \mathrm{g} \mathrm{mL}^{-1}$ for $8 \mathrm{~h}$ incubation. For longer incubation times, the metabolic activities decrease from $25 \mu \mathrm{g} \mathrm{mL}^{-1}$ onwards.

Furthermore, the cell toxicity of CQDs-1 and CQDs-2 was established on HeLa and U-87 MG cell lines (Figure S7a). All CQDs are non-toxic to HeLa and U-87 MG cells until $100 \mu \mathrm{g} \mathrm{mL}$ ${ }^{1}$ for both incubation times. HeLa and U-87 MG cells were incubated with CQDs at $100 \mu \mathrm{g} \mathrm{mL}$ ${ }^{1}$ during $24 \mathrm{~h}$ and then, nuclei were stained with Hoechst 33258, a fluorescent dye for labeling DNA in fluorescence microcopy (Figure S7b). The green fluorescence, which is attributed to the CQDs, is homogeneously distributed in the cytoplasm after $24 \mathrm{~h}$ when incubated at $37{ }^{\circ} \mathrm{C}$, 
which confirms the adsorption and internalization of positively charged CQDs- 2 inside the cells, while there is almost no fluorescence for CQDs-1.
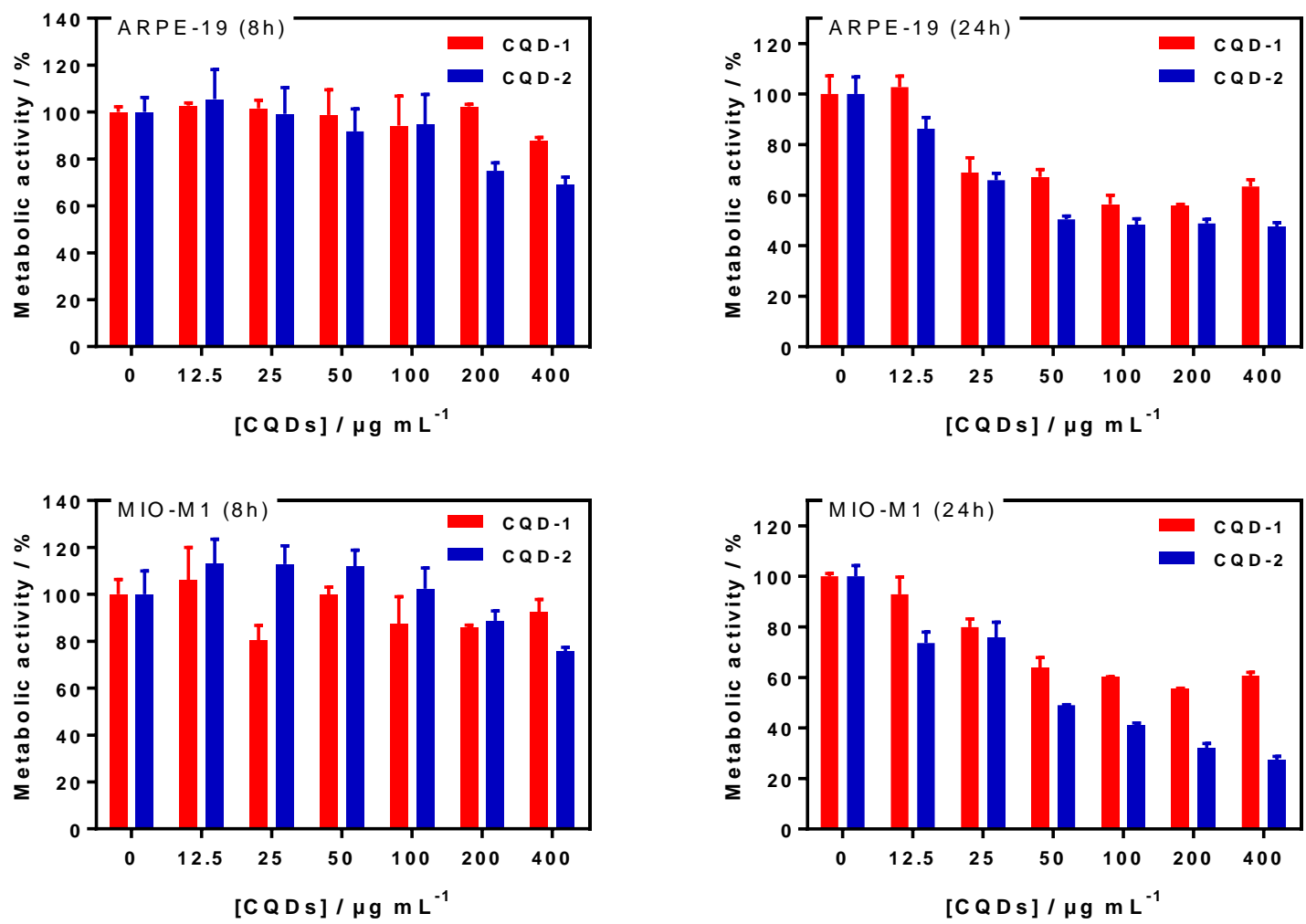

Figure 5: Metabolic activity of CQDs. Metabolic activity of ARPE-19 and MIO-M1 cells treated with CQDs: cells were grown in 96-well plates $\left(10^{4}\right.$ cells/well) with $100 \mu \mathrm{L}$ of culture medium containing increasing concentration of CQDs for $8 \mathrm{~h}$ (left) and $24 \mathrm{~h}$ (right). The results, expressed as percentage of viability, are the mean value of two independent experiments with each treatment performed in triplicate. Negative control: without CQDs.

\subsection{Inhibition of type I collagen aggregation with CQD-1 and CQD-2}

Fibril formation of type I collagen has been widely studied by some research teams. ${ }^{40-46}$ Therefore, type I collagen was investigated in this present work to assess to what extent CQDs may inhibit its fibrillation. As explained in the experimental section, fibril formation of type I collagen was initiated by incubating collagen I solution at $37{ }^{\circ} \mathrm{C}$. The change in the optical density of the sample at $313 \mathrm{~nm}$ as a function of time was recorded (Figure 6a). After a lag time of about $2 \mathrm{~min}$, a collagen self-assembly growth phase was observed, reaching a plateau after about 3 min which is in line with other reports. ${ }^{45}$ AFM imaging (Figure 6b) confirmed the formation of collagen fibers, with a typical diameter of $\sim 150 \mathrm{~nm}$. 
The presence of a low amount of negatively charged CQD-1 $\left(<100 \mu \mathrm{g} \mathrm{mL} \mathrm{L}^{-1}\right)$ mainly influences the lag time i.e. the onset of collagen fibrillation. Through AFM imaging aggregate formation is observed in the presence of $100 \mu \mathrm{g} \mathrm{mL}^{-1}$ of CQD-1, with fibers with a diameter of $40-50 \mathrm{~nm}$, much smaller compared with control collagen fibers. A higher CQD-1 concentration of $250 \mu \mathrm{g}$ $\mathrm{mL}^{-1}$ resulted in complete inhibition of the collagen fibrillation. In contract, the presence of a low amount of positively charged CQD-2 induced an acceleration of type I collagen fibril formation (Figure 6a). The AFM image of collagen in the presence of $100 \mu \mathrm{g} \mathrm{mL}^{-1} \mathrm{CQD}-2$ (Figure 6b) indicates a quasi-complete inhibition of fibrillation with isolated aggregates of 1.5 $\mu \mathrm{m}$ in length and a diameter of $\sim 50 \mathrm{~nm}$. Note that a complete inhibition of collagen fibrillation was already observed at a concentration of $150 \mu \mathrm{g} \mathrm{mL}^{-1}$.

At low CQDs concentration, some collagen fibril assembly remains. This indicates that the inhibitory effect of CQDs on the nucleation step and intermolecular interactions is concentration dependent. CQDs inhibits fibrillation in a mechanism involving an endothermic reaction along with electrostatic and hydrophobic interactions between neighboring molecules. Hydrogen bonds are believed to be the most stabilizing forces prevailing in these proteins. It is, thus, hypothesized that the hydrogen bonds may be formed between carbonyl and carboxylic acid groups present on the surface of CQD-1 and amine and hydroxyl groups of type I collagen. ${ }^{47}$ This might alter the interactive forces between collagen molecules. The main differences between both type of particles is their surface charge and functionalization. Note that physiochemical properties of nanoparticles such as size and surface charge, have been considered as critical factors for their antifibrotic properties. ${ }^{48,49}$

(a)
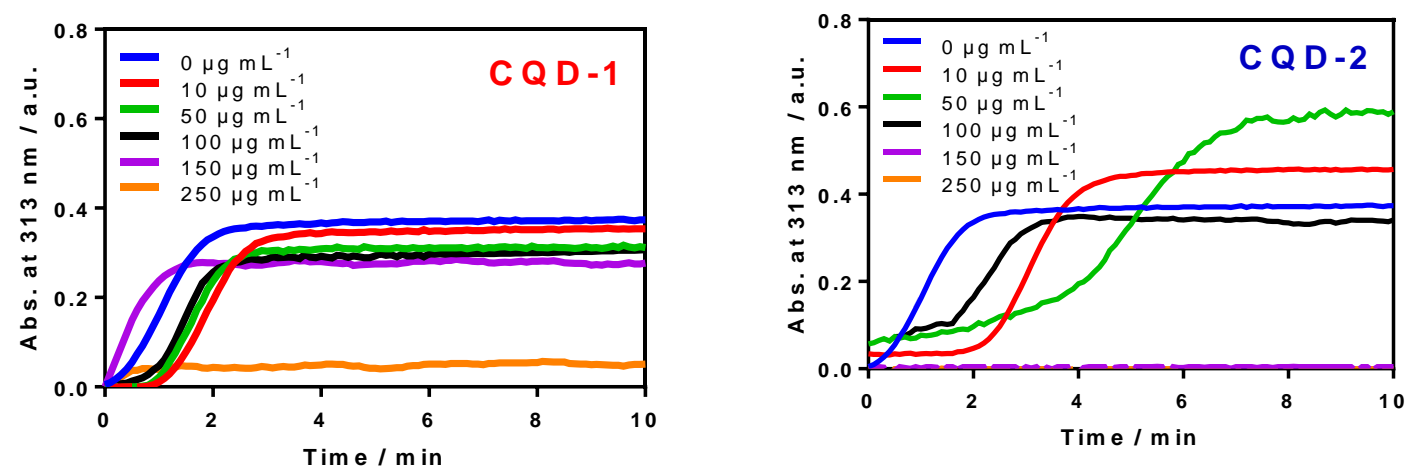

(b) 

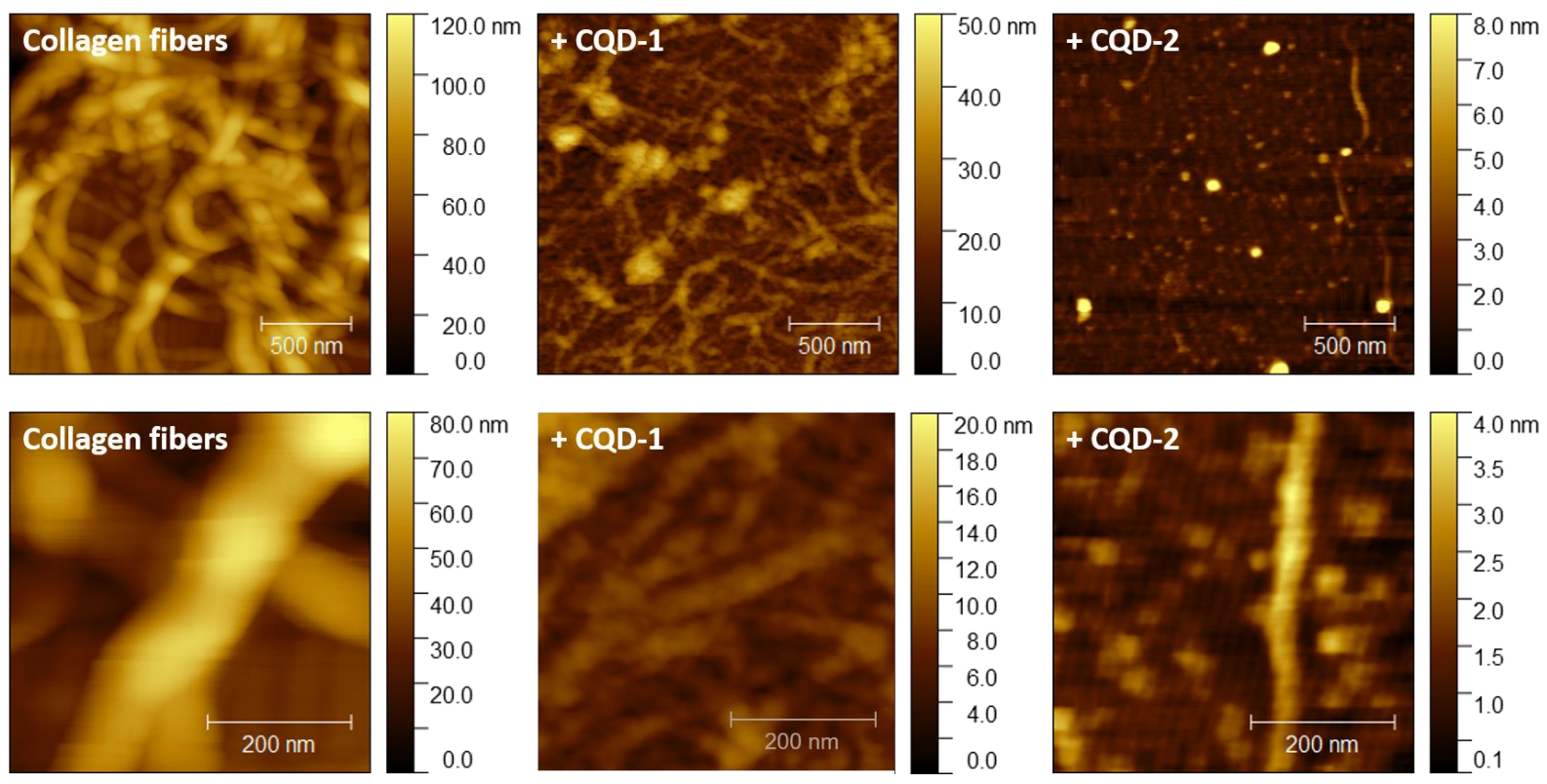

Figure 6: Collagen I fibril formation and inhibition by CQDs. (a) Absorption (at $313 \mathrm{~nm}$ ) over time of a collagen I solution $\left(0.3 \mathrm{mg} \mathrm{mL}^{-1}\right)$ incubated at $37^{\circ} \mathrm{C}$ in the absence and presence of increasing concentrations of CQD-1 and CQD-2; (b) AFM images of collagen I ( $0.3 \mathrm{mg} \mathrm{mL}-$ ${ }^{1}$ ) in the absence and presence of $100 \mu \mathrm{g} \mathrm{mL}^{-1} \mathrm{CQD}-1$ and CQD-2.

\subsection{Destruction of type I collagen fibers under pulsed-laser irradiation in the presence of CQDs}

We have recently shown that Au NPs, able to trigger the formation of vapor nanobubbles (VNBs) under pulsed-laser irradiation, adhere to type I collagen fibers and can destroy vitreous opacities. ${ }^{17}$ Though, different to CQDs, Au NPs do not seem to interfere with collagen fibrillation (Figure S8). The destruction of the type I collagen fibers/vitreous opacities is believed to be mechanical: collapsing VNBs induce a mechanical force which breaks down the fibers. To investigate whether a similar effect could be observed with CQD-1 and CQD-2, type I collagen fibers were mixed with CQD-1 or CQD-2 and irradiated with a pulsed-laser at different fluences and wavelengths. Dark field microscopy was used to visualize the collagen fibers before and after laser irradiation.

As seen in Figure 7a, applying light fluences of 1.1 and $4.5 \mathrm{~J} / \mathrm{cm}^{2}$ and laser irradiation wavelengths of 488, 561 and $650 \mathrm{~nm}$, without the use of CQDs, did not destroy the type I collagen fibers. Irradiation of the collagen fibers in the presence of CQD-1 showed the same result: the collagen fibers remained intact (Figure 7b). This contrasts to the use of CQD-2 (Figure 7c). Light pulses of $<3 \mathrm{~ns}$ at 488 and $561 \mathrm{~nm}\left(4.5 \mathrm{~J} \mathrm{~cm}^{-2}\right)$ clearly destroyed the collagen fibers. Using a higher wavelength of $650 \mathrm{~nm}$ did no longer allow the complete destruction of 
the fibers, even if 100 pulses were applied (Figure 7c). This could be expected as CQD-2 hardly absorbs at $650 \mathrm{~nm}$ (Figure 3a). Given that the $488 \mathrm{~nm}$ laser light might be (more) harmful for ocular tissues (if compared to $561 \mathrm{~nm}$ light), we selected $561 \mathrm{~nm}$ to continue our experiments on human opacities.

(a)

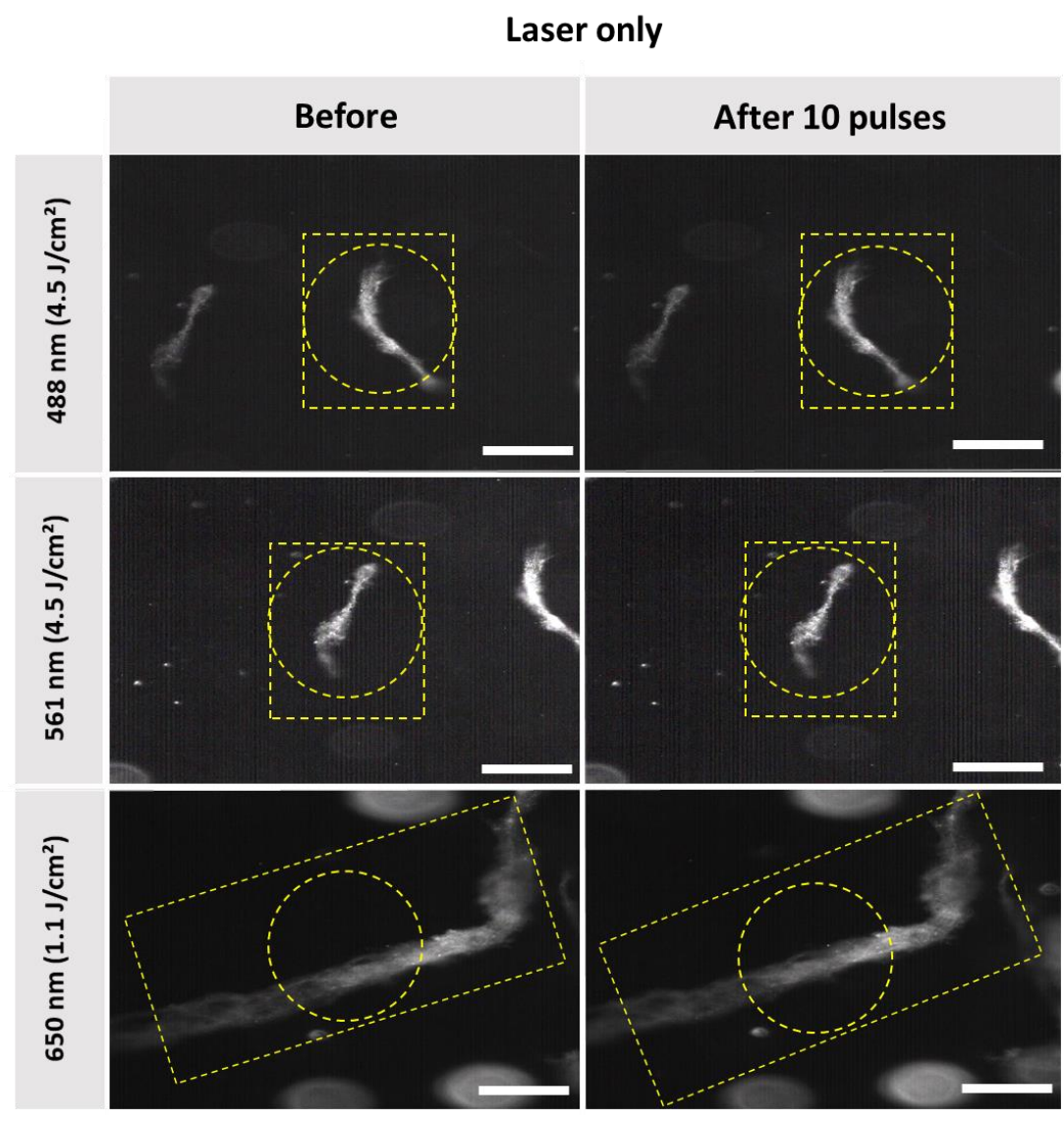


(b)

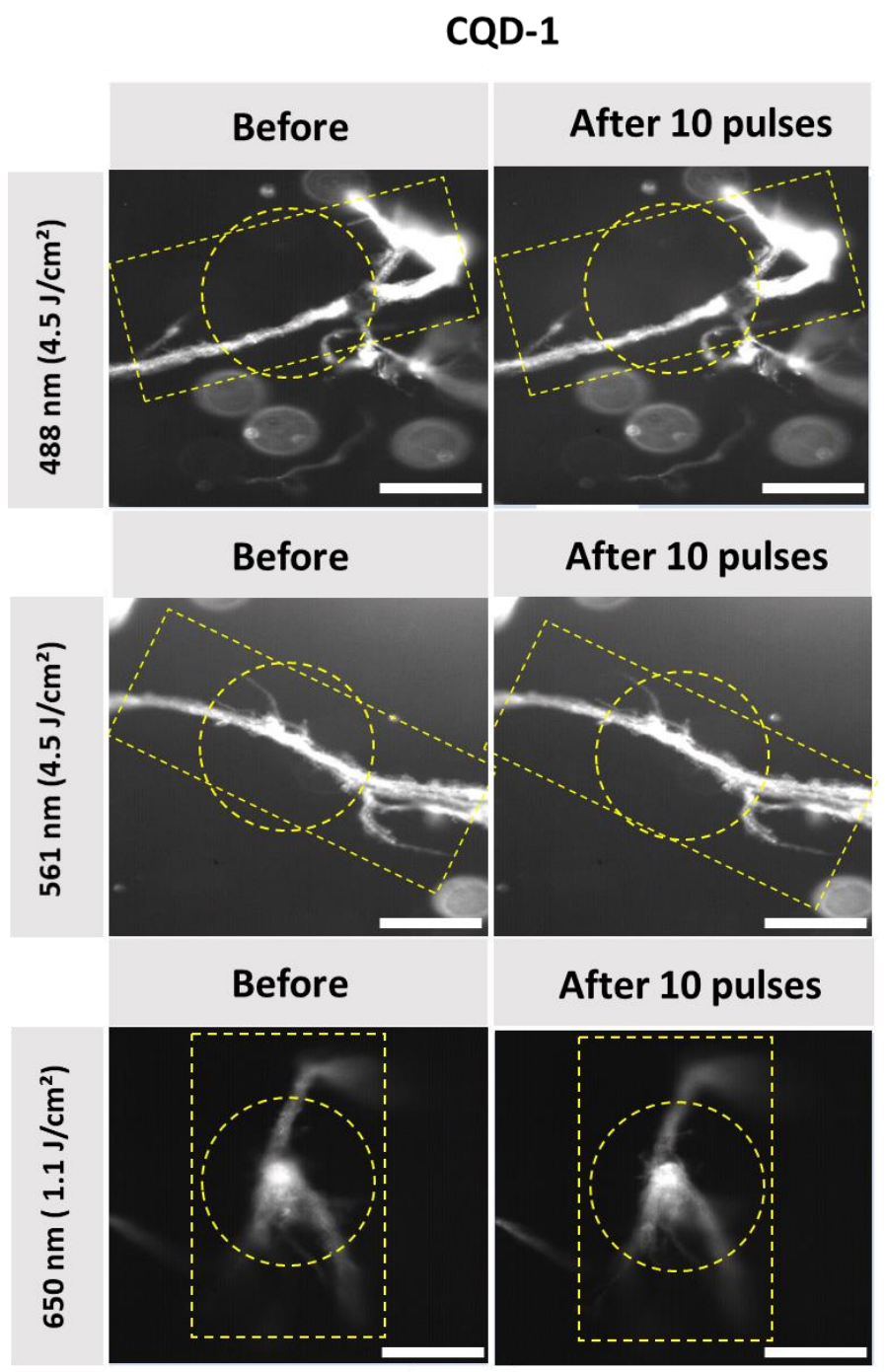


(c)

\section{CQD-2}

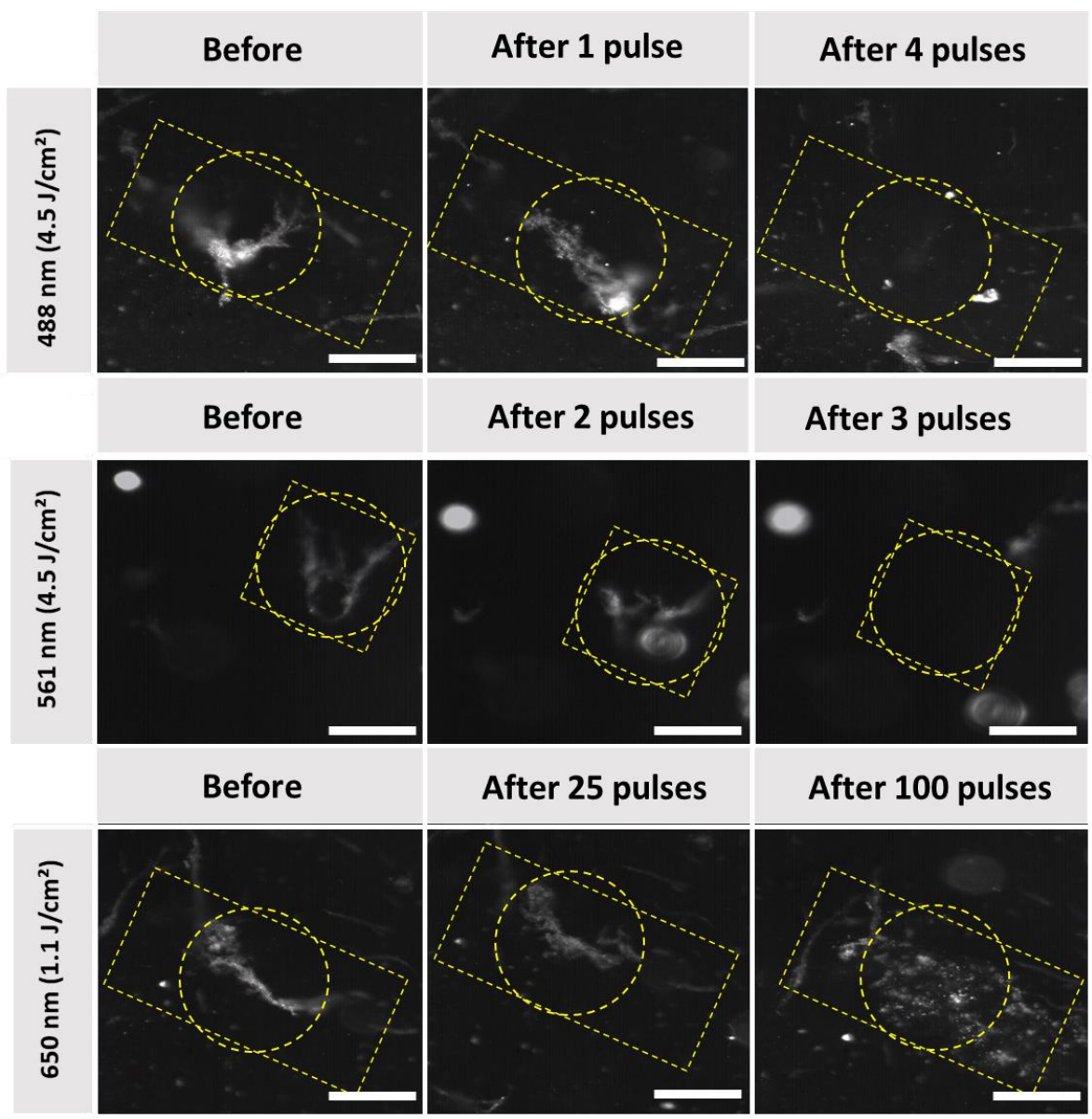

Figure 7: Dark-field microscopy images of water-dispersed type I collagen fibers. (a)

Before and after light illumination with lasers of different intensities and wavelengths (488, 561 and $650 \mathrm{~nm}$ ). (b) Before and after light illumination with lasers of different intensities and wavelengths $(488,561$ and $650 \mathrm{~nm})$ in the presence of negatively charged CQD-1 $\left(0.4 \mathrm{mg} \mathrm{mL}^{-}\right.$ $\left.{ }^{1}\right)$. (c) Before and after light illumination with lasers of different intensities and wavelengths $(488,561$ and $650 \mathrm{~nm})$ in the presence of positively CQD-2 $\left(0.4 \mathrm{mg} \mathrm{mL}^{-1}\right)$. Scale bar: $100 \mu \mathrm{m}$. The yellow dotted squares/rectangles indicate the position of the targeted fiber. Dotted circles indicate the position of the laser beam.

While we find that the pulsed-laser irradiation in the presence of CQD-2 totally destroys the aggregates, the same approach is inefficient using CQD-1. This difference may rely on the surface charge of the two nanoparticles, which is negative for CQD-1 and positive for CQD-2 (Table 1). The difference in charges could lower the interaction of CQD-1 with the collagen fibers, when compared to CQD-2. Temperature effects could be also involved. However, CQDs show no photothermal activity (Figure 4). In spite one can expect higher photothermal effects with gold nanoparticles, no differences in terms of fluence was observed for fiber destruction: 
in both cases fluence of $4.5 \mathrm{~J} / \mathrm{cm}^{2}$ was required for efficient fiber demolition. This can be explained by the relatively small size of both AuNPs and CQDs which cannot trigger formation of vapor nanobubbles in solution but only when they form clusters or in situation where the distance between particles is reduced (as it is likely the case when both AuNPs or CQDs are bound to fibers).

However, inhibition of the fibrillation with both types of CQDs clearly suggests that they can both interact with collagen molecules (Figure 7a).

\subsection{Assessment of the mobility of CQDs in vitreous by fluorescence recovery after photobleaching (FRAP)}

After intravitreal injection, CQDs must keep their mobility to diffuse and their capacity to bind to the target, the vitreous opacities. Although we expected that CQDs, due to their relatively small size (around $10 \mathrm{~nm}$ ), could diffuse in the bovine vitreous, ${ }^{50}$ we decided to perform FRAP experiments to ensure they were mobile and could therefore reach the opacities after intravitreal injection. Indeed, while CQDs are positively charged and the vitreous net charge is negative, one can expect that they will interact with the glycosaminoglycans and collagen network of the vitreous thus forming aggregates. FRAP is a technique allowing to measure the diffusion coefficients of fluorescent molecules or nanoparticles. Briefly, a laser of strong intensity is applied on the sample and induces its photobleaching. The diffusion of the surrounding (fluorescent) molecules (or particles) into the bleached area can be measured and observed by a progressive increase of fluorescence intensity. Recovery curves can, therefore, be obtained from which diffusion coefficients can be calculated.

As observed in confocal images (Figure 8a), fluorescence recovery curves in water and in vitreous (Figure 8b and c) indicate that a complete fluorescence recovery occurs in the bleached area. This observation attests that cationic CQDs can diffuse both in water and in bovine vitreous. Their diffusion coefficients in water and vitreous are comparable $(31.4+/-7.4$ $\mu \mathrm{m}^{2} / \mathrm{s}$ and $32.9+/-13.2 \mu \mathrm{m}^{2} / \mathrm{s}$, respectively). Our observation suggests that, despite a positive charge, CQDs keep their mobility in vitreous. The diffusion of CQDs can be due to their small size (around $10 \mathrm{~nm}$ ) allowing diffusion through the meshes of the collagen network whose size are comprised between 500 and $1000 \mathrm{~nm} .{ }^{50}$ This result also indicates that, after intravitreal injection, CQD-2 can sufficiently diffuse to bind the opacities. 
a)

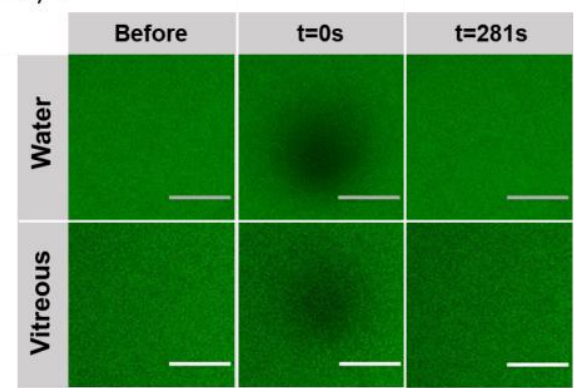

b)

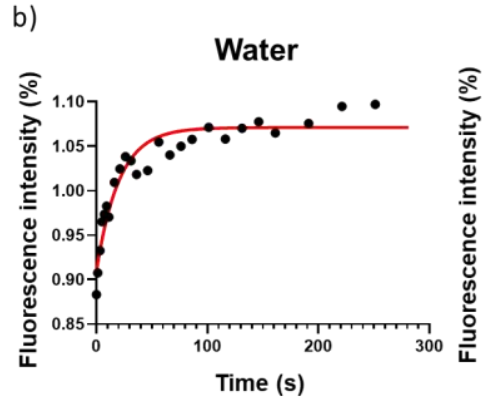

c)

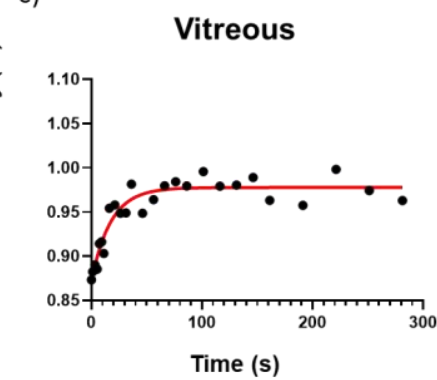

Figure 8: Diffusion of cationic CQDs. (a) Confocal microscopy images of CQDs (2.2 $\mathrm{mg} \mathrm{mL}^{-}$

$\left.{ }^{1}\right)$ before, during $(\mathrm{t}=0 \mathrm{~s})$ and $130 \mathrm{~s}$ after photobleaching in water and in bovine vitreous. In water (b) and in vitreous (c) fluorescence of recovery as a function of time occurs in the bleaching area indicating that the cationic CQDs have the ability to diffuse both in water and in bovine vitreous. Scale bar $=100 \mu \mathrm{m}$.

\subsection{Destruction of human vitreous opacities treated with CQD-2 under pulsed-laser irradiation}

Type I collagen fibers were previously used to attest the efficacy of their destruction after treatment with CQD-2 and upon pulsed-laser irradiation. Since the collagen composition, size and shape of vitreous opacities differ from type I collagen fibers, we therefore decided to test our concept on real opacities from human vitreous obtained from patients after vitrectomy. The first observation is that, without treatment with CQDs, no destruction or change in opacities could be observed after 50 pulses at $561 \mathrm{~nm}$ using a fluence of $4.5 \mathrm{~J} / \mathrm{cm}^{2}$, suggesting the laser itself cannot destroy the opacities. However, as shown in Figure 9, when vitreous samples were mixed with CQD-2 (0.4 mg mL $\left.\mathrm{m}^{-1}\right)$ a clear destruction of the opacities could be observed after 50 pulses $\left(561 \mathrm{~nm} ; 4.5 \mathrm{~J} / \mathrm{cm}^{2}\right)$. This result is in line with previous observations from some of us at a same wavelength and fluence using $10 \mathrm{~nm}$ hyaluronan-coated Au NPs and suggests a similar efficacy. ${ }^{17}$ 

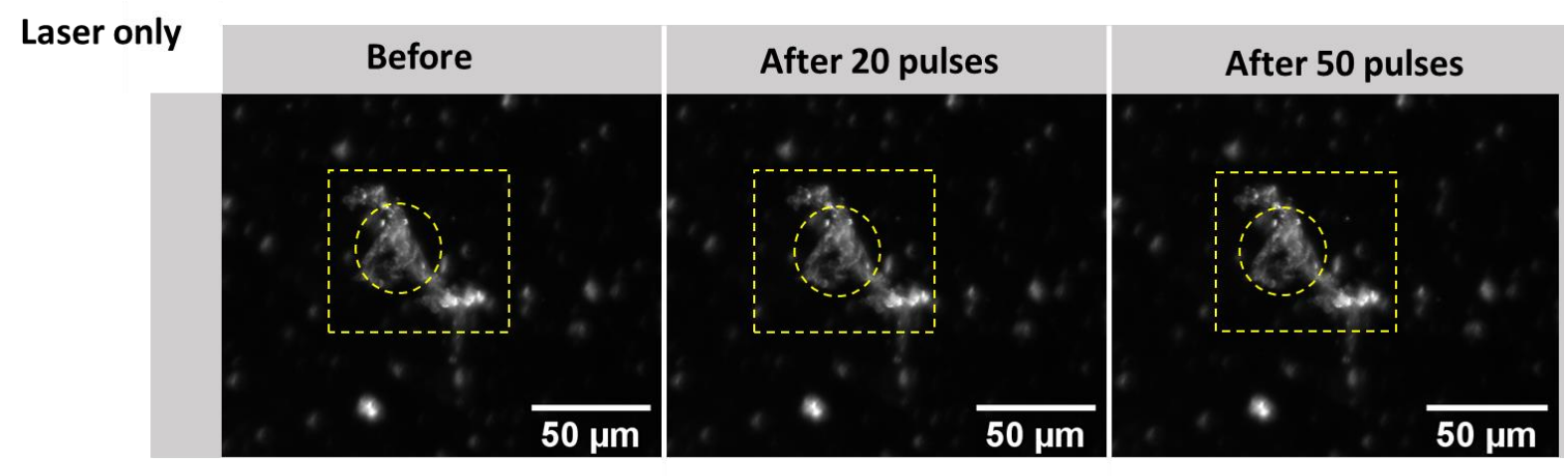

CQDs-2
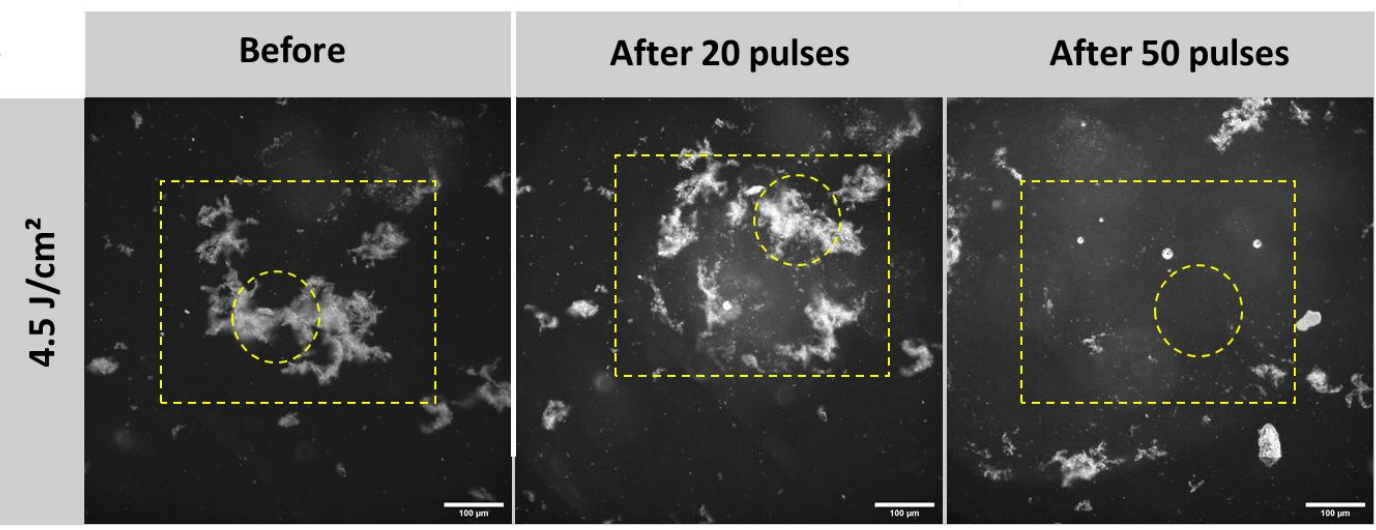

Figure 9: Dark-field microscopy images of human vitreous opacities: Before and after light illumination (20 and 50 pulses) with laser $\left(561 \mathrm{~nm}, 4.5 \mathrm{~J} \mathrm{~cm}^{-2}\right)$ in absence or in presence of CQDs-2 (0.4 mg mL $\mathrm{m}^{-1}$ ). Scale bar (for bottom row): $100 \mu \mathrm{m}$. The yellow dotted squares/rectangles indicate the position of the targeted fiber. Dotted circles indicate the position of the laser beam.

\section{Conclusion}

In this work, we have shown that cationic CQDs could inhibit the fibrillation of type I collagen in vitro and destroy type I collagen fibers and human vitreous opacities through the generation of light-induced VNBs at a low fluence $\left(4.5 \mathrm{~J} / \mathrm{cm}^{2}\right)$. While negatively charged CQDs can inhibit collagen fibrillation, they were unable to destroy the collagen fibers at the studied fluences and wavelengths. Interestingly, while Au NPs can generate VNBs and have been reported to destroy vitreous opacities, they did not impact collagen fibrillation. CQDs can promote the destruction of vitreous opacities in a similar manner (fluence, number of pulses) with a capacity to inhibit collagen fibrillation. Thanks to this dual effect (i.e. inhibition and destruction), cationic CQDs become attractive therapeutic tools for the treatment of vitreous opacities by avoiding or postponing their reformation after laser treatment. 
Taken together, our results suggest that this dual effect provided by CQDs could be further explored in the context of fibrotic diseases and more generally for diseases involving protein aggregation.

\section{Supporting Information Available}

Figure S1: TEM images of CQD-1 (a) and CQD-2 (b)

Figure S2: XPS high resolution spectra of CQD-1 and CQD-2.

Figure S3: Kaiser test for the determination of the presence of primary amine groups: (a) Calibration curve (benzylamine solution from 10 to $100 \mu \mathrm{M}$ ). (b) UV-vis absorption spectra of CQD-2, heated at $100{ }^{\circ} \mathrm{C}$ during $10 \mathrm{~min}$, cooled down on ice and further diluted with $1 \mathrm{~mL}$ of ethanolic solution $(\mathrm{EtOH} /$ water=60:40).

Figure S4: (a) Zeta potential analysis. (b) Photographs of CQDs suspensions $\left(250 \mu \mathrm{g} \mathrm{mL}^{-1}\right)$ after $1 \mathrm{~h}$ incubation at $37^{\circ} \mathrm{C}$ in water (W), PBS (0.01 M, P) and Dulbecco's Modified Eagle's medium (M).

Figure S5: The relationship between the integrated fluorescence intensity and the absorbance of quinine sulfate (QS), CQD-1 and CQD-2.

Figure S6: Optical stability of CQD-1 (red) and CQD-2 (blue) in various conditions: Fluorescence emission after 2-years, normalized fluorescence emission after 2-years and relative fluorescence emission of CQDs solution $\left(20 \mu \mathrm{g} \mathrm{mL}^{-1}\right.$ in PBS) during UV light irradiation at $360 \mathrm{~nm}\left(10 \mathrm{~mW} \mathrm{~cm}^{-2}\right)$ during 3 hours.

Figure S7: Metabolic activity and CQDs uptake: (a) Metabolic activity of HeLa and U-87 MG cells treated with CQDs: cells were grown in 96-well plates $\left(10^{4}\right.$ cells/well $)$ with $100 \mu \mathrm{L}$ of culture medium containing increasing concentration of CQDs for $8 \mathrm{~h}$ (left) and $24 \mathrm{~h}$ (right). The results, expressed as percentage of viability, are the mean value of two independent experiments with each treatment performed in triplicate. Negative control: without CQDs. (b) Fluorescence microscopy of HeLa and U-87 MG cells treated with $400 \mu \mathrm{g} \mathrm{mL}^{-1}$ of CQDs for $24 \mathrm{~h}$ The blue signal corresponds to the nuclei stained with Hoechst 33258, while the green signal is attributed to CQDs. Scale bars $=100 \mu \mathrm{m}$.

Figure S8: Fibril formation study of type I collagen using turbidity data: UV-vis absorption evolution of collagen $\left(0.3 \mathrm{mg} \mathrm{mL}^{-1}\right)$ at $313 \mathrm{~nm}$ in PBS buffer (pH 7.4) at $37{ }^{\circ} \mathrm{C}$ under static condition (blue) and in the presence of increasing concentrations of gold nanoparticles (AuNPs) $\left(10^{11}-10^{13}\right.$ AuNPs $\left.\mathrm{mL}^{-1}\right)$.

\section{Acknowledgements}


Financial support from the Centre National de la Recherche Scientifique (CNRS), the University of Lille is acknowledged. This work was partly supported by the French Renatech network. The authors would also like to acknowledge funding from the European Union's Horizon 2020 research and innovation program under grant agreement No 810685 (DelNam project).

\section{Experimental section}

\subsection{Materials}

Glucosamine hydrochloride, ethylenediamine and $\beta$-alanine were purchased from Sigma Aldrich Chimie (France) and used as-received.

Samples of human vitreous containing opacities were collected at the VMR Institute for Vitreous Macula Retina (Huntington Beach, CA, USA) from patients undergoing vitrectomy for the treatment of vision-degrading myodesopsia. The study protocol adhered to the Declaration of Helsinki. Prior to surgery, patients gave a written informed consent that has been reviewed and accepted by the ethical committee of Saint Joseph Health Center for clinical research (Irvine, CA, USA). After vitrectomy, the (undiluted) samples were frozen and stored at $-80{ }^{\circ} \mathrm{C}$ until further use.

\subsection{Synthesis of carbon quantum dots (CQDs) and gold nanoparticles (AuNPs)}

\subsubsection{Synthesis of CQD-1}

Carboxylated CQDs were synthesized according to a modified procedure. ${ }^{32}$ To a stirring solution of glucosamine hydrochloride $(1.00 \mathrm{~g}, 4.63 \mathrm{mmol})$ in distilled water $(20 \mathrm{~mL})$ in a 30 $\mathrm{mL}$ vial G30, $\beta$-alanine ( $0.454 \mathrm{~g}, 1.1$ eq.) was added and stirred to ensure homogeneity. The reactor was then placed in a microwave (Monowave 450, Anton-Parr). The solution was heated under pressure for $2 \mathrm{~min}$ from room temperature to $200{ }^{\circ} \mathrm{C}$ and then maintained for $1 \mathrm{~min}$ at $200{ }^{\circ} \mathrm{C}$ under stirring $(1200 \mathrm{rpm})$. The resulting brown solution was centrifuged at $4{ }^{\circ} \mathrm{C}(10000$ $\mathrm{g}, 10 \mathrm{~min}$ ) in order to remove big particles. The supernatant was dialyzed against water during $24 \mathrm{~h}$ (SpectraPor RC membranes, $1 \mathrm{kDa}$ ) and then kept (or lyophilized) to yield a brown solution.

\subsubsection{Synthesis of CQD-2}

To a stirring solution of glucosamine hydrochloride $(1.00 \mathrm{~g}, 4.63 \mathrm{mmol})$ in distilled water (20 $\mathrm{mL}$ ) in a $30 \mathrm{~mL}$ vial G30, ethylenediamine (0.307 g, 1.1 eq.) was added and stirred to ensure homogeneity. The reactor was then placed in a microwave (Monowave 450, Anton-Parr). The 
solution was heated under pressure for 5 min from room temperature to $200{ }^{\circ} \mathrm{C}$ and then maintained for $10 \mathrm{~min}$ at $200{ }^{\circ} \mathrm{C}$ under stirring (1200 rpm). The resulting brown solution was centrifuged at $+4{ }^{\circ} \mathrm{C}(10000 \mathrm{~g}, 10 \mathrm{~min})$ in order to remove big particles. The supernatant was dialyzed against water during $24 \mathrm{~h}$ (SpectraPor RC membranes, $1 \mathrm{kDa}$ ) and then kept (or lyophilized) to yield a brown solution.

\subsubsection{Synthesis of hyalorunic acid-functionalized gold nanoparticles (AuNPs)}

The synthesis and hyaluronic acid (HA)-coated AuNPs (10 nm) was performed in house, as previously reported. ${ }^{17}$ Briefly, $\mathrm{HAuCl}_{4}$ (in water) and sodium ascorbate (in water) were mixed at equimolar ratios (concentration $=0.2 \mathrm{mM}$, final volume $=100 \mathrm{~mL}$ ) under rapid stirring for at least $1 \mathrm{~h}$. The formed AuNPs were washed by centrifugation (10 min at $13000 \mathrm{~g}$ ), resuspended in an aqueous solution of HA (2-4 pM), and kept under rotation overnight. HA-coated AuNPs were finally washed by centrifugation, resuspended in water, and stored for further use. The synthetized NPs were characterized by DLS and zeta potential (Zetasizer Nano, Malvern) measurements. The final concentration of the AuNPs was estimated from the extinction of the dispersions as measured at the maximal extinction wavelength (Ext) and the theoretical extinction cross section $\sigma_{\text {EXT }}\left(\mathrm{cm}^{2}\right)$, as previously reported. ${ }^{17}$

\subsection{Primary amine quantification using a modified Kaiser test}

The amount of primary amine groups on the CQDs was calculated using a spectrophotometric assay based on the modified Kaiser test with the following modifications. ${ }^{51}$ Briefly, CQDs-2 were diluted to $0.1 \mathrm{mg} \mathrm{mL}^{-1}$ in milli-Q water. To this solution was added $50 \mu \mathrm{L}$ of phenol solution ( $40 \mathrm{~g}$ in $10 \mathrm{~mL} \mathrm{EtOH}$ ), $50 \mu \mathrm{L}$ of potassium cyanide ( $2 \mathrm{~mL}$ of $\mathrm{KCN}$ solution $-65 \mathrm{mg}$ in $100 \mathrm{~mL}$ water - in $100 \mathrm{~mL}$ pyridine) and $50 \mu \mathrm{L}$ of ninhydrin solution (2.5 g in $50 \mathrm{~mL}$ EtOH). The mixture was heated at $100{ }^{\circ} \mathrm{C}$ during $10 \mathrm{~min}$, cooled down on ice and further diluted with $1 \mathrm{~mL}$ of ethanolic solution $(\mathrm{EtOH} /$ water=60/40). The absorption of the mixture was determined in triplicate at $570 \mathrm{~nm}$ using a Perkin Elmer Lambda UV/Vis 950 dual-beam spectrophotometer in $10 \mathrm{~mm}$ quartz cuvette. Benzylamine solution was used from 10 to $100 \mu \mathrm{M}$ to generate the calibration curve.

\subsection{Characterizations}

Atomic Force Microscopy (AFM): AFM images were recorded in tapping mode on a Dimension $^{\mathrm{TM}} 3100$ (Digital Instruments) in air at a scan rate of $1 \mathrm{~Hz}$, and with an antimony doped $n$-type silicon cantilever (resistivity: $0.01-0.025 \mathrm{Ohm} . \mathrm{cm}$, frequency resonance ranging 
from 130 to $250 \mathrm{kHz}$ - with a constant stiffness $\mathrm{k} \sim 48 \mathrm{~N} \cdot \mathrm{m}^{-1}$ ). Samples were diluted by 15 with filtered PBS, deposited on freshly cleaved mica substrates, dried in air and then rinsed three times with water. Data were analyzed with a Gwyddion software (2.56).

Transmission electron microscopy (TEM): The morphology and sizes of CQDs were characterized using FEI Tecnai G2-20 microscope.

$U V$-visible measurements: Absorption spectra were recorded from 200 to $800 \mathrm{~nm}$ using a Perkin Elmer Lambda UV/Vis 950 dual-beam spectrophotometer in $10 \mathrm{~mm}$ quartz cuvette. CQDs were diluted to $20 \mu \mathrm{g} \mathrm{mL}^{-1}$ in PBS (pH 7.4, 0.01 M, Gibco®).

Raman spectroscopy: Raman spectroscopy measurements were performed on a LabRam HR Micro-Raman system (Horiba Jobin Yvon) using a $473 \mathrm{~nm}$ laser diode as excitation source. CQDs were deposited on a clean silicon wafer. The visible light is focused by a $100 \times$ objective. The scattered light is collected from 100 to $4000 \mathrm{~cm}^{-1}$ by the same objective in backscattering configuration, dispersed by a $1800 \mathrm{~mm}$ focal length monochromator and detected by a CCD camera.

Fourier transform infrared (FT-IR) spectroscopy: FT-IR analyses were performed on Thermo Fisher Scientific Inc. Nicolet 8700, in the frequency range between 650 and $4000 \mathrm{~cm}^{-1}$ with a resolution of $6 \mathrm{~cm}^{-1}$. Dried CQDs (around $1 \mathrm{mg}$ ) were mixed with potassium bromide powder (200 mg) in an agar mortar. The mixture was pressed into a pellet under 7 tons of load for 10 min, and the spectrum was recorded immediately. An average of 64 scans was carried out for each sample.

$X$-ray photoelectron spectroscopy (XPS): XPS spectra were recorded on a VG Escalab 220XL spectrometer from Vacuum Generators featuring a monochromatic A1 K $\alpha$ X-ray source (1486.6 $\mathrm{eV}$ ) and a spherical energy analyzer operated in the CAE (constant analyzer energy) mode $(\mathrm{CAE}=100 \mathrm{eV}$ for survey spectra and $\mathrm{CAE}=40 \mathrm{eV}$ for high-resolution spectra $)$, using the electromagnetic lens mode. The angle between the incident X-rays and the analyzer is $58^{\circ}$ and the detection angle of the photoelectrons is $30^{\circ}$.

DLS measurements: The average hydrodynamic diameter and the zeta-potential were recorded on a Zetasizer® Nano ZS (Malvern Instruments S.A., Worcestershire, UK). All the batches were diluted to $200 \mu \mathrm{g} \mathrm{mL}^{-1}$ in milli-Q water and analyzed in triplicate at $20{ }^{\circ} \mathrm{C}$. The zeta potential of CQDs in milli-Q water $\left(200 \mu \mathrm{g} \mathrm{mL}^{-1}\right)$ was measured at $20{ }^{\circ} \mathrm{C}$ using a folded zeta capillary cell (DTS1070). Experiments consisted of 15 runs per measurement with an applied voltage of $150 \mathrm{~V}$.

Fluorescence measurements: Emission and excitation fluorescence spectra were recorded between 200-900 nm using a Xenius XC spectrofluorometer (Safas S.A., Monaco). 
Fluorescence measurements of CQDs were performed in PBS (pH 7.4, 0.01 M, Gibco®) at 20 $\mu \mathrm{g} \mathrm{mL}^{-1}$ (excitation and emission bandwidth $=10 \mathrm{~nm}$, step=1 nm, PMT voltage $=460 \mathrm{~V}$, ultrascan). For quantum yield measurements, fluorescence emissions were recorded from 350$900 \mathrm{~nm}$ with an excitation wavelength of $350 \mathrm{~nm}$ (excitation and emission bandwidth=10 nm, step $=1 \mathrm{~nm}$, PMT voltage $=260 \mathrm{~V}$, ultrascan). Integrated fluorescence emissions were calculated from 365 to $680 \mathrm{~nm}$.

\subsection{Measurement of the photothermal properties}

The photothermal properties of $50 \mu \mathrm{g} \mathrm{mL}^{-1}$ solutions of CQDs in PBS ( $\mathrm{pH}$ 7.4, 0.01 M, Gibco®) were determined by using a continuous laser beam at $810 \mathrm{~nm}$ (Gbox model, Fournier Medical Solution) at two power densities $\left(1\right.$ and $\left.2 \mathrm{~W} \mathrm{~cm}^{-2}\right)$. The temperature changes were recorded by an infrared camera (FLIR A655sc, IR lens: $\mathrm{f}=24.6 \mathrm{~mm}$ and $25^{\circ}$ ) and an image analysis software FLIR ResearchIR Max4.

\subsection{Biological evaluations}

Cell viability assay

The ARPE-19 spontaneously immortalized cell line of human retinal pigment epithelium, the MIO-M1 Müller progenitor cell line, the HeLa cell line derived from cervical carcinoma from a 31-year-old female (ECACC 93021013, Sigma Aldrich Chimie S.a.r.l, St. Quentin Fallavier, France) and the U-87 MG cell line derived from a malignant glioma (ECACC 89081402, Sigma Aldrich Chimie S.a.r.1, St. Quentin Fallavier, France) were cultured and maintained in Dulbecco's Modified Eagle's medium (DMEM, Gibcoß) high glucose with 2 mM Glutamine, DMEM/F12 media with $2 \mathrm{mM}$ Glutamine and DMEM supplemented with $10 \%$ fetal bovine serum (FBS, Gibco®) and 1\% penicillin-streptomycin (Gibco®), respectively, in a humidified incubator at $37{ }^{\circ} \mathrm{C}$ and $5 \% \mathrm{CO}$. Cells were seeded at a density of $10^{4}$ cells/well in a 96-well plate and grown for $24 \mathrm{~h}$ before assay. The culture medium was replaced with a fresh medium that contains the CQDs from 0 to $400 \mu \mathrm{g} \mathrm{mL}^{-1}$. After $24 \mathrm{~h}$, the old medium was aspirated and cells were washed with PBS. The cell viability was evaluated using resazurin cell viability assay. Briefly, $100 \mu \mathrm{L}$ of the resazurin solution $\left(11 \mu \mathrm{g} \mathrm{mL}^{-1}\right)$ in appropriate media were added to each well and the plate was incubated for $4 \mathrm{~h}$ in the humidified incubator. The fluorescence emission of each well was measured at $593 \mathrm{~nm}$ (20-nm bandwidth) with an excitation at $554 \mathrm{~nm}(18-\mathrm{nm}$ bandwidth) using a Cytation ${ }^{\mathrm{TM}} 5$ Cell Imaging Multi-Mode Reader (BioTek Instruments SAS, France). Each condition was replicated three times and the mean fluorescence value of nonexposed cells was taken as $100 \%$ cellular viability. 


\section{Cell imaging}

HeLa and U-87 MG cells were seeded in a 96-well plate at a density of $10^{4}$ cells/well and grown for $24 \mathrm{~h}$ before assay. The CQDs solution were diluted in DMEM/10\% FBS to a final concentration of $400 \mu \mathrm{g} \mathrm{mL}^{-1}$. After 24 -hours incubation at $37^{\circ} \mathrm{C}$, the cell monolayers were washed three times with PBS and then stained with Hoechst $33258\left(5 \mu \mathrm{g} \mathrm{mL}^{-1}\right)$ in PBS in the dark for $5 \mathrm{~min}$. The plates were directly observed using a Cytation ${ }^{\mathrm{TM}} 5$ Cell Imaging MultiMode Reader (BioTek Instruments SAS, France) equipped with a 20x objective (Plan Fluorite WD 6.7 NA 0.45). The fluorescence signal was acquired using excitation and emission filter sets for DAPI (exc. 377/50 nm and em. 447/60 nm) and for GFP (exc. 469/35 nm and em. $525 / 39 \mathrm{~nm}$ ). All the images were further analyzed by Gen5iPlus 3.04 Imaging Software.

\subsection{Collagen fibril formation}

Collagen I from rat tail (A10483-01, Gibco®) was dissolved in PBS (pH 7.4, 0.01 M, Gibco®) at $0{ }^{\circ} \mathrm{C}$ (ice bath) to obtain a final concentration of $0.3 \mathrm{mg} \mathrm{mL}^{-1}$. CQDs at different concentrations were added. Subsequently, the collagen/CQDs solution was homogenized and incubated without stirring during $1 \mathrm{~h}$ at $37{ }^{\circ} \mathrm{C}$. The fibrillation process was followed by measuring the absorbance/turbidity of the solutions at $313 \mathrm{~nm}$ by using a NanoDrop ${ }^{\mathrm{TM}}$ One $\mathrm{C}$ spectrophotometer (Thermo Scientific). The baseline correction was done just before to start each experiment $(\mathrm{t}=0 \mathrm{~min})$.

\subsection{Photoablation of collagen fibers and human vitreous opacities}

Photoablation experiments were performed on collagen fibers in water and human vitreous containing opacities. Collagen fibers $\left(0.02 \mathrm{mg} \mathrm{mL}^{-1}\right)$ were mixed with CQDs in water $(0.4 \mathrm{mg}$ $\mathrm{mL}^{-1}$ ) and allowed to equilibrate for $30 \mathrm{~min}$ before the experiment. After thawing the human vitreous samples, they were directly mixed with an equal volume of a dispersion of CQDs (typically $0.4 \mathrm{mg} \mathrm{mL}^{-1}$ in water). The samples were then allowed to equilibrate for $30 \mathrm{~min}$ at room temperature prior to applying (nanosecond) laser pulses.

First, dark-field microscopy imaging was performed to locate and align the nanosecond laser on the collagen fibers or human vitreous opacities in the sample. To generate vapor nanobubbles, $7 \mathrm{~ns}$ laser pulses were used. The wavelengths of the laser light were $488 \mathrm{~nm}, 561 \mathrm{~nm}$ or $650 \mathrm{~nm}$. A beam expander (\#GBE05-A, Thorlabs) combined with an iris diaphragm (\#D37SZ, Thorlabs) was used to adjust the diameter of the laser beam to $150 \mu \mathrm{m}$. The laser pulse energy was 
monitored by an energy meter (J-25MB-HE\&LE, Energy Max-USB/RS sensors, Coherent) synchronized with the pulsed laser.

\subsection{Diffusion experiments in water and bovine vitreous by photobleaching}

Fluorescence recovery after photobleaching (FRAP) experiments were performed to determine the mobility of the fluorescent CQDs in water and bovine vitreous. FRAP experiments were performed using a confocal scanning laser microscope (Nikon A1R) equipped with a $10 \times$ lens (CFI Plan Apochromat; Nikon, Badhoevedorp, The Netherlands). In a FRAP experiment, fluorescent nanoparticles in a (vitreous) sample are irreversibly bleached in a small area (100 $\mu \mathrm{m})$ by an intense laser beam. The surrounding particles (i.e. outside of the bleached zone) will diffuse into the photobleached area, leading to a fluorescence recovery over time. Diffusion coefficients (D) can be derived from the rate and amount of recovery of fluorescence in the bleached zone by fitting the data points to appropriate equations. ${ }^{52}$

For FRAP experiments in water, $5 \mu \mathrm{L}$ of a water solution of CQD-2 $\left(2.2 \mathrm{mg} \mathrm{mL}^{-1}\right)$ was applied between a microscope slide and a coverslip which were separated by a $120 \mu \mathrm{m}$-thick double adhesive spacer (Secure-Seal Spacer; Molecular Probes, Leiden, The Netherlands). For FRAP experiments in bovine vitreous, bovine vitreous was carefully removed from the eye ball (to avoid undesired liquefaction) and $400 \mu \mathrm{L}$ were carefully cut and transferred into a glassbottomed dish. $40 \mu \mathrm{L}$ of a solution of CQD-2 $\left(2.2 \mathrm{mg} \mathrm{mL}^{-1}\right)$ were then injected in the vitreous using a $1 \mathrm{~mL}$ syringe equipped with a $21.5 \mathrm{G}$ needle and allowed to equilibrate at room temperature for $30 \mathrm{~min}$ before performing FRAP experiments. 


\section{References}

1. L. Vaughan, M. Mendler, S. Huber, P. Bruckner, K. H. Winterhalter, M. I. Irwin and R. Mayne, J. Cell Biol. , 1988, 106, 991-997.

2. P. N. Bishop, Prog. Retin. Eye Res. , 2000, 19, 323-344.

3. P. N. Bishop, D. F. Holmes, K. E. Kadler, D. McLeod and K. J. Bos, Invest. Ophthalmol. Vis. Sci. 2, 2004, 45, 1041-1046.

4. J. Sebag, Prog. Reti. Eye Res., 2020, 100847.

5. J. Sebag and E. Balazs, Invest. Ophthalmol. Vis. Sci., 1989, 30, 1867-1871.

6. L. Los, R. van der Worp, M. van Luyn and J. Hooymans, Invest. Ophthalmol. Vis. Sci., 2003, 44, 2828-2833.

7. G. Garcia, M. Khoshnevis, J. Nguyen-Cuu, K. M. P. Yee, J. H. Nguyen, A. A. Sadun and J. Sebag, Graefe's Arch Clin Exp Ophthalmol 2018, 256, 919-925.

8. J. Sebag, Am. J. Ophthalmol., 2011, 152, 3-4.

9. J. Mamou, C. A. Wa, K. M. Yee, R. H. Silverman, J. A. Ketterling, A. A. Sadun and J. Sebag, Invest. Ophthalmol. Vis. Sci., 2015, 56, 1611-1617.

10. E. Ankamah, J. Sebag, E. Ng and J. M. Nolan, Antioxidants 2020, 9, 7.

11. J. Sebag, K. M. P. Yee, L. Huang, C. Wa and A. A. Sadun, Retina, 2014, 34, $1062-$ 1068 .

12. J. Sebag, K. M. P. Yee, J. H. Nguyen and J. Nguyen-Cuu, Ophthalmology Retina, 2018, 2, 881-887.

13. R. Milston, M. Optoma and M. C. Madigan, Survey of ophthalmology, 2016, 61, 211227.

14. J. H. Nguyen, J. Nguyen-Cuu, F. Yu, K. M. Yee, J. Mamou, R. H. Silverman, J. Ketterling and J. Sebag, Ophthalmology Retina, 2019, 126, 1517-1526.

15. P. Stalmans, M. S. Benz, A. Gandorfer, A. Kampik, S. Pakola and J. A. Haller, N Engl J Med., 2012, 367, 606e615.

16. J. Sebag, Retina, 2009, 29, 871-874.

17. F. Sauvage, J. C. Fraire, K. Remaut, J. Sebag, K. Peynshaert, M. Harrington, F. Van de Velde, R. Xiong, M.-J. Tassignon, T. Brans, K. Braeckmans and S. De Smedt, ACS Nano 2019, 13, 8401-8416.

18. K. Nekoueian, M. Amiri, M. Sillanpää, F. Marken, R. Boukherroub and S. Szunerits, Chem. Soc. Rev., 2019, 48, 4281-4316

19. M.-X. Zhao and B.-J. Zhu, Nanoscale Res. Lett., 2016, 11, 207.

20. A. Barras, Q. Pagneux, F. Sane, Q. Wang, R. Boukherroubs, H. Didier and S. Szunerits, ACS Appl. Mater. Interfaces 2016, 8, 9004-9013.

21. H.-J. Jian, R.-S. Wu, T.-Y. Lin, Y.-J. Li, H.-J. Lin, S. G. Harroun, J.-Y. Lai and C.-C. Huang, ACS Nano, 2017, 11, 6703-6716.

22. A. Shoval, A. Markus, Z. Zhou, X. Liu, R. Cazelles, I. Willner and Y. Mandel, Small, 2019, 15, 1902776.

23. D. Qu, X. Miao, X. Wang, C. Nie, Y. Li, L. Luo and e. al., J. Mater. Chem. B, 2017, 5, 4988-4992.

24. B. B. Karakoçak, J. Liang, S. Kavadiya, M. Y. Berezin, P. Biswas and N. Ravi, ACS Appl. Nano Mater. , 2018, 1, 3682-3692.

25. C.-Q. Li, X.-Y. Liu, S.-L. Li, P. Jiang, F.-J. Jiang and Y. Liu, ACS Appl. Bio Mater., 2019, 2, 4067-4076.

26. X. Han, Z. Jing, W.-C. Wu, B. Zou, Z. Peng, P. Ren, A. Wikramanayake, Z. Lu and R. M. Leblanc, Nanoscale, 2017, 9, 12862.

27. S. Li, L. Wang, C. C. Chusuei, V. M. Suarez, P. L. Blackwelder, M. Micic, J. Orbulesu and R. M. Leblanc, Chem. Mater., 2015, 27, 1764-1771. 
28. Y. Q. Q., J. C. Jin, J. Q. Zhang, B. B. Wang and Y. Liu, J. Mater. Chem. B, 2017, 5, 2010-2018.

29. L. Wang, S. Zhu, T. Lu, G. Zhang, J. Xu, Y. Song, Y. Li, L. Wang, B. Yang and F. Li, J. Mater. Chem. B, 2016,4, 4913-4921 2016.

30. A. Łoczechin, K. Séron, A. Barras, E. Giovanelli, S. Belouzard, Y.-C. Chen, MetzlerNolte, N. , R. Boukherroub, J. Dubuisson and S. zunerits, ACS Appl. Mater. Interfaces, 2019.

31. T. V. de Medeiros, J. Manioudakis, F. Noun, J.-R. Macairan, F. Victoria and R. Naccache, J. Mater. Chem. C, 2019, 7, 7175-7195

32. S. A. Hill, D. Benito-Alifonso, S. A. Davis, D. J. Morgan, M. Berry and M. C. Galan, Sci. Rep., 2018, 8, 12234

33. S. K. Tammina and Y. Yang, J. Photoch. Photobiol. A, 2020, 387, 112134.

34. S. Chandra, S. Pradhan, S. Mitra, P. Patra, A. Bhattacharya, P. Pramanik and A. Goswami, Nanoscale, 2014, 6, 3647-3655.

35. J.-B. Wu, M.-L. Lin, X. Cong, H.-N. Liu and P.-H. Tan, Chem. Soc. Rev., 2018, 47, 1822-1873.

36. P. Zhu, K. Tan, Q. Chen, J. Xiong and L. Gao, Chem. MAter., 2019, 31, 4732-4742.

37. W. Zhang, Y. Wang, X. Liu, X. Meng, H. Xu, Y. Xu, B. Liu, X. Fang, H.-B. Li and T. Ding, Phys. Chem. Chem Phys., 2017, 19, 28653-28665.

38. H. Peng and J. Travas-Sejdic, Chem. Mater., 2009, 21, 5563-5565.

39. S. Zhu, Y. Song, X. Zhao, J. Shao, J. Zhang and B. Yang, Nano Research, 2015, 8, 355-381.

40. H.-J. Cjung, A. Steplewski, K. Y. Chung, J. Uitto and A. Fertala, J. Biol. Chem., 2008, 283, 25879-25889.

41. X. Yin, L. Zhao, S.-g. Kang, J. Pan, Y. Song, M. Zhang, G. Xing, F. Wang, J. Li, R. Zhou and Y. Zhao, Nanoscale, 2013, 5, 7341.

42. K. Rasheeda and N. Fathima, Colloids Surf. B 2018, 170, 273-279.

43. H. Bharathy and N. Nishad Fathima, Int. J. Biol. Macromol . 2017, 10, 179-186.

44. J. Jayamania, R. Ravikanth Reddy, B. Madhand and G. Shanmugama, Int. J. Biol. Macromol . 2018, 107, 175-185.

45. B. G. Anand, K. Dubey and D. S. Shekhawat, Langmuir, 2017, 33, 13252-13261.

46. S. Perumal, K. Dubey, R. Badhwar, K. J. George, R. K. Sharma, G. Bagler, B. Madhan and K. C. Kar, Eur. Biophys. J., 2015, 44, 69-76.

47. H. S. Frederick, W. F. Joseph and P. S. Gurinder, J. Biomech. , 2003, 36, 1529-1553.

48. Y. Kim, J.-H. Park, H. Lee and J.-M. Nam, Sci. Rep., 2016, 6, 1.

49. F. Sauvage, J. Schymkowitz, F. Rousseau, B. Z. Schmidt, K. Remaut, K. Braeckmans and S. C. De Smedt, Nano Today, 2020, 31, 100837.

50. S. Xu, N. J. Boylan, J. S. Suk, Y.-Y. Wang, E. A. Nance, J.-C. Yang, P. J. McDonnell, R. A. Cone, E. J. Duh and J. Hanes, J. Control. Release, 2013, 167, 76-84.

51. G. Jarre, S. Heyer, E. Memmel, T. Meinhardt and A. Krueger, Beilstein Journal of Organic Chemistry, 2014, 10, 2729-2737.

52. T. K. L. Meyvis, S. S. De-Smedt, P. Van-Oostveldt and J. Demeester, Pharm Res. , 1999, 16, 1153-1162. 